\title{
Fourier 积分算子的局部光滑性及其相关研究
}

献给陈恕行教授 80 华诞

高传伟 ${ }^{1}$ ，苗长兴 ${ }^{2 *}$

1. 北京大学北京国际数学研究中心, 北京 100871 ;

2. 北京应用物理与计算数学研究所, 北京 100088

E-mail: cwgao@pku.edu.cn, miao_changxing@iapcm.ac.cn

收稿日期: 2020-06-03; 接受日期: 2020-11-02；网络出版日期: 2021-03-05; * 通信作者

国家自然科学基金 (批准号: 11926303 和 11831004) 和博士后基金 (批准号: 8206300279) 资助项目

摘要 本文综述满足电影型 (cinematic) 曲率条件的 Fourier 积分算子的局部光滑性及其相关研究. 电 影型曲率条件包含非退化条件及曲率条件. 作为范例重点讨论如何通过双线性方法建立变系数版本的 平方函数不等式, 进而改进了 Mockenhaupt-Seeger-Sogge 局部光滑性的结果. 与此同时, 本文还分析了 解决局部光滑性猜想的困难、可能的途径, 以及它与其他数学猜想之间的联系.

关键词 Fourier 积分算子 局部光滑性估计 平方函数不等式 双线性方法 电影型曲率条件

MSC (2020) 主题分类 $35 \mathrm{~S} 30,35 \mathrm{~L} 15$

\section{1 背景简介}

Fourier 积分算子 (Fourier integral operators, FIOs) 起源于拟微分算子, 拟微分算子来源于构造椭 圆方程的拟基本解, 它是研究椭圆型方程的基本工具 (参见文献 $[1,2]$ ). 而 Fourier 积分算子与构造强 双曲方程的拟基本解紧密相关, 这方面的工作至少可以追溯到 Lax ${ }^{[3]}$. Fourier 积分算子的主旨是在余 切从框架下研究双曲型方程解的奇性传播和 FIOs 的局部光滑性等相关问题. 作为 Fourier 积分算子 的典型范例, Euclid 空间的半波算子局部光滑性猜想是许多著名数学猜想的高级形式, 它直接意味着 Bochner-Riesz 猜想、限制性猜想和 Kakeya 猜想等, 体现了偏微分方程与调和分析、几何测度论和数 论等不同数学领域之间的内在联系. 经过 Hörmander ${ }^{[4]}$ 的奠基性工作以及 Stein ${ }^{[5,6]}$ 等众多数学家的 发展, Fourier 积分算子已经成为变系数 (或非平坦) 调和分析的核心. 特别是平方函数估计与变系数 分离性 (decoupling) 理论的发展, 为研究以局部光滑性猜想为代表的一系列数学猜想提供了有力工具. 本文重点综述一类满足电影型 (cinematic) 曲率条件的 Fourier 积分算子的局部光滑性的研究进展, 分 析局部光滑性猜想的困难及解决该猜想可能的途径. 与此同时, 讨论它与其他数学猜想之间的内在联 系. 关于 FIOs 理论的奠基性工作, 可参见文献 $[4,7]$. 有关 FIOs 及相关的偏微分方程的基本理论, 可 参见文献 [8-14].

英文引用格式: Gao C W, Miao C X. Local smoothness of Fourier integral operators and related research (in Chinese). Sci Sin Math, 2021, 51: 847-880, doi: 10.1360/SSM-2020-0173 


\subsection{Fourier 积分算子的定义}

为了引入 FIOs, 考虑两个典型范例.

例 1.1 FIOs 的原型来源于下面的半波算子:

$$
\mathrm{e}^{\mathrm{i} t \sqrt{-\Delta}} f(x):=(2 \pi)^{-\frac{n}{2}} \int_{\mathbb{R}^{n}} \mathrm{e}^{\mathrm{i}(x \xi+t|\xi|)} \hat{f}(\xi) d \xi .
$$

假设函数 $f_{0}$ 和 $f_{1}$ 满足适当的正则性, 记

$$
f_{+}:=\frac{1}{2}\left(f_{0}-\mathrm{i}(\sqrt{-\Delta})^{-1} f_{1}\right), \quad f_{-}:=\frac{1}{2}\left(f_{0}+\mathrm{i}(\sqrt{-\Delta})^{-1} f_{1}\right),
$$

则

$$
u(x, t):=\mathrm{e}^{\mathrm{i} t \sqrt{-\Delta}} f_{+}(x)+\mathrm{e}^{-\mathrm{i} t \sqrt{-\Delta}} f_{-}(x)
$$

是波动方程 Cauchy 问题

$$
\left\{\begin{array}{l}
\left(\partial_{t}^{2}-\Delta\right) u=0, \\
u(x, 0)=f_{0}(x), \quad \partial_{t} u(x, 0)=f_{1}(x)
\end{array}\right.
$$

的解. 容易看出, (1.2) 右边的两项均可表示成如下形式:

$$
\mathscr{F}_{t} f(x):=(2 \pi)^{-\frac{n}{2}} \int_{\mathbb{R}^{n}} \mathrm{e}^{\mathrm{i}(x \xi \pm t|\xi|)}|\xi|^{-j} \hat{f}(\xi) d \xi, \quad j=0,1 .
$$

例 1.2 设 $(M, g)$ 为 $n$ 维 Riemann 紧流形, $\Delta_{g}$ 是 Laplace-Beltrami 算子, 则 $-\Delta_{g}$ 具有非负离 散谱, 满足 (特征根的重数也计算在内)

$$
0=\lambda_{0}^{2}<\lambda_{1}^{2} \leqslant \lambda_{2}^{2} \leqslant \lambda_{3}^{2} \leqslant \cdots .
$$

因此,

$$
-\Delta_{g}=\sum_{j=0}^{\infty} \lambda_{j}^{2} E_{j}
$$

其中 $E_{j}$ 为投影算子, 它把 $L^{2}(M)$ 中的函数投影到特征根 $\lambda_{j}^{2}$ 所对应的特征空间上, 详见文献 $[15,16]$. 定义流形 $(M, g)$ 上的半波算子

$$
\mathrm{e}^{\mathrm{i} t \sqrt{-\Delta_{g}}} f:=\sum_{j=0}^{\infty} \mathrm{e}^{\mathrm{i} t \lambda_{j}} E_{j} f .
$$

若 $f_{0}$ 和 $f_{1}$ 为定义在 $M$ 上的函数, 用 $-\Delta_{g}$ 代替 $-\Delta$, 类似于例 1.1 定义的 $f_{+}$和 $f_{-}$, 则

$$
u(x, t):=\mathrm{e}^{\mathrm{i} t \sqrt{-\Delta_{g}}} f_{+}(x)+\mathrm{e}^{-\mathrm{i} t \sqrt{-\Delta_{g}}} f_{-}(x)
$$

为方程

$$
\left\{\begin{array}{l}
\left(\partial_{t}^{2}-\Delta_{g}\right) u=0, \\
u(x, 0)=f_{0}(x), \quad \partial_{t} u(x, 0)=f_{1}(x)
\end{array}\right.
$$

的解. 在局部坐标下, 可以构造 (1.5) 的拟基本解, 即对某一时刻 $t_{0}$ 和所有的 $0<t<t_{0}$, 存在相函数 $\phi$ 和零阶象征 $a$ 使得

$$
\mathrm{e}^{\mathrm{i} t \sqrt{-\Delta_{g}}} f(x):=\int_{\mathbb{R}^{n}} \mathrm{e}^{\mathrm{i} \phi(x, t, \xi)} a(x, t, \xi) \hat{f}(\xi) d \xi+R_{t} f(x),
$$

其中 $R_{t}$ 为光滑算子, 即具有快速衰减象征的拟微分算子 (参见文献 [17]). 
由上述两个例子, 可以诱导出如下经典 FIOs 的定义:

定义 1.1 (Fourier 积分算子的定义) 设 $\mu \in \mathbb{R}, f \in \mathcal{S}\left(\mathbb{R}^{n}\right)$, 定义 $\mu$ 阶 Fourier 积分算子为

$$
\mathscr{F} f(x):=\int_{\mathbb{R}^{n}} \mathrm{e}^{\mathrm{i} \phi(x, \xi)} a(x, \xi) \hat{f}(\xi) d \xi,
$$

其中 $\phi$ 和 $a$ 分别满足

(i) 相函数 $\phi: \mathbb{R}^{n} \times \mathbb{R}^{n} \rightarrow \mathbb{R}$ 关于变量 $\xi$ 是 1- 次齐函数, 且在 $\xi=0$ 之外光滑;

(ii) 振幅 $a(x, \xi) \in S^{\mu}$, 即 $a \in C^{\infty}\left(\mathbb{R}^{n} \times \mathbb{R}^{n} \backslash\{0\}\right)$, 且满足对 $(\alpha, \beta) \in \mathbb{N}_{0}^{n} \times \mathbb{N}_{0}^{n}$, 有

$$
\left|\partial_{x}^{\beta} \partial_{\xi}^{\alpha} a(x, \xi)\right| \lesssim \alpha, \beta(1+|\xi|)^{\mu-\alpha} .
$$

注 1.1 注意到 (1.8) 中定义的 FIOs 的核函数具有形式

$$
K(x, y)=\int_{\mathbb{R}^{n}} \mathrm{e}^{-\mathrm{i}(\langle y, \xi\rangle-\phi(x, \xi))} a(x, \xi) d \xi,
$$

由此启发我们用振荡积分所决定的分布来定义 FIOs. 称光滑相函数 $\varphi(w, \theta): W \times \mathbb{R}^{N} \backslash\{0\} \rightarrow \mathbb{R}$ 是非 退化的, 如果它满足下面的条件:

(i) $\varphi$ 关于变量 $\theta$ 是 1 - 次齐次函数;

(ii) 对所有 $(w, \theta) \in W \times \mathbb{R}^{N} \backslash\{0\}$, 均有 $\nabla_{w, \theta} \varphi(w, \theta) \neq 0$;

(iii) 如果 $\partial_{\theta} \varphi(w, \theta)=0$, 则

$$
\bigwedge_{j=1}^{N} \nabla_{w, \theta} \partial_{\theta_{j}} \varphi(w, \theta) \neq 0 .
$$

对非退化相函数 $\varphi$ 和光滑象征 $a \in S^{\mu}\left(W \times \mathbb{R}^{N}\right)$, 定义

$$
I[\varphi, a](w):=\int_{\mathbb{R}^{N}} \mathrm{e}^{\mathrm{i} \varphi(w, \theta)} a(w, \theta) d \theta, \quad w \in W .
$$

从而可以得到 FIOs 定义的局部形式.

定义 1.2 称连续线性算子 $\mathscr{F}: C_{c}^{\infty} \rightarrow \mathcal{D}^{\prime}(X)$ 为局部 Fourier 积分算子, 如果其 Schwartz 核函 数是由某个齐次振荡积分 $I[\varphi, a]$ 给出的, 其中相函数 $\varphi: X \times Y \times \mathbb{R}^{N} \backslash\{0\} \rightarrow \mathbb{R}$ 为非退化的, 象征 $a \in S^{\mu}\left(X \times Y \times \mathbb{R}^{N}\right)$. 设 $X, Y \subset \mathbb{R}^{n}$, 给定实验函数 $f \in C_{c}^{\infty}(Y)$, 在分布意义下, $\mathscr{F}$ 可表示为

$$
\mathscr{F} f(x)=\int_{\mathbb{R}^{n}} \int_{\mathbb{R}^{N}} \mathrm{e}^{\mathrm{i} \varphi(w, \theta)} a(w, \theta) d \theta f(y) d y, \quad x \in X .
$$

注 1.2 局部 FIOs 定义的缺点是不同 $(\varphi, a)$ 可能定义相同的振荡积分 $I[\varphi, a]$, 这样我们自然要 问: 是否存在不依赖于 $[\varphi, a]$ 选取的定义方式? 答案是肯定的, 这就是整体 FIOs 的定义. 这种定义必 求助于 FIOs 对应的 Schwartz 核函数奇性的几何特性, 从而导致了 FIOs 对应的典则关系构造. 根据 Hörmander 相函数的等价定理, FIOs 单纯依赖典则关系, 不依赖特定 $[\varphi, a]$ 的选择, 详见文献 $[4,7,17]$. 需要说明的是, 整体 Fourier 积分算子在局部坐标下可表现为 (1.8) 中的形式.

\section{2 电影型曲率条件}

引入电影型曲率条件之前, 考察例 1.1 中的相函数 $\phi(x, \xi)=x \xi+t|\xi|$. 它显然满足

$\left(\mathrm{H}_{1}\right) \operatorname{rank} \partial_{z \xi}^{2} \phi(z, \xi)=n$; 
$\left(\mathrm{H}_{2}\right)$ 定义 Gauss 映射 $G(z, \xi):=\frac{G_{0}(z, \xi)}{\left|G_{0}(z, \xi)\right|}$, 其中

$$
G_{0}(z, \xi):=\bigwedge_{i=1}^{n} \partial_{\xi_{i}} \partial_{z} \phi(z, \xi)
$$

曲率条件

$$
\left.\operatorname{rank} \partial_{\xi \xi}^{2}\left\langle\partial_{z} \phi(z, \xi), G\left(z, \xi_{0}\right)\right\rangle\right|_{\xi=\xi_{0}}=n-1 .
$$

例 1.2 中的半波算子 $\mathrm{e}^{\mathrm{i} t \sqrt{-\Delta_{\mathrm{g}}}}$ 在局部坐标意义下可以写成

$$
\mathrm{e}^{\mathrm{i} t \sqrt{-\Delta_{g}}} f \cong \int \mathrm{e}^{\mathrm{i} t \phi(x, t, \eta)} a(x, t, \eta) \hat{f} d \eta,
$$

其中相函数 $\phi(x, t, \eta)$ 同样满足条件 $\left(\mathrm{H}_{1}\right)$ 和 $\left(\mathrm{H}_{2}\right)$.

一般地, 设 $n \geqslant 2, Z$ 和 $Y$ 为两个光滑紧流形, 其中 $Z$ 和 $Y$ 分别满足 $\operatorname{dim} Z=n+1, \operatorname{dim} Y=n$. 通过典则关系 $\mathscr{C}$ 定义的整体 Fourier 积分算子记为 $\mathscr{F} \in I^{\mu-\frac{1}{4}}(Z, Y ; \mathscr{C})$. 在辛结构 $d \zeta \wedge d z-d \eta \wedge d y$ 下, $\mathscr{C}$ 为 $T^{*} Z \backslash 0 \times T^{*} Y \backslash 0$ 上的齐次锥性质的 $2 n+1$ 维闭 Lagrange 子流形. 对给定一点 $z_{0} \in Z$, 考 虑自然投影算子图

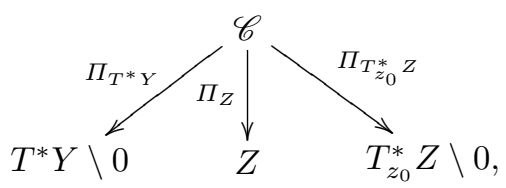

其中 $\Pi_{T^{*} Y} 、 \Pi_{T_{z_{0}}^{*} Z}$ 及 $\Pi_{Z}$ 是分别从 $\mathscr{C}$ 到 $T^{*} Y \backslash 0 、 T_{z_{0}}^{*} Z \backslash 0$ 及 $Z$ 的投影. 电影型曲率条件描述如下:

非退化条件 (1.11) 中前两个投影是非退化的, 满足

$$
\begin{aligned}
& \operatorname{rank} d \Pi_{T^{*} Y} \equiv 2 n, \\
& \operatorname{rank} d \Pi_{Z} \equiv n+1 .
\end{aligned}
$$

即说明投影映射是淹没.

曲率条件 $\Gamma_{z_{0}}=\Pi_{T_{z_{0}}^{*} Z}(\mathscr{C})$ 为余切空间 $T_{z_{0}}^{*} Z \backslash 0$ 的浸入超曲面.

注意到 $\mathscr{C}$ 的齐次性质, 作为 (1.12) 和 (1.13) 的直接结果, 推出 $\Gamma_{z_{0}}$ 是 $T_{z_{0}}^{*} Z \backslash 0$ 的 $n$ 维光滑雉形 超曲面.

锥条件 对任意的 $\zeta \in \Gamma_{z_{0}}, \Gamma_{z_{0}}$ 存在 $n-1$ 个非零主曲率.

称 $\mathscr{C}$ 满足电影型曲率条件, 如果它满足非退化条件、曲率条件和雉条件. 容易看出, 在局部坐标 意义下, 非退化、曲率和雉条件可以解析表达成 $\left(\mathrm{H}_{1}\right)$ 和 $\left(\mathrm{H}_{2}\right)$.

\section{3 相函数的标准形式}

利用局部化和平移方法, 不妨假设 $\operatorname{supp} a \subset Z \times \Xi$, 其中 $Z=X \times T, X \subset B_{1}(0) \subset \mathbb{R}^{n}, T \subset(-1,1)$ 均是包含原点的小邻域, $\Xi \subset \Gamma_{1}$, 其中 $\Gamma_{1}$ 是以 $e_{n}=(0, \ldots, 0,1) \in \mathbb{R}^{n}$ 为中心的小扇形邻域. 在上述归 结下, 存在合适局部坐标系, 使得满足条件 $\left(\mathrm{H}_{1}\right)$ 和 $\left(\mathrm{H}_{2}\right)$ 的相函数 $\phi(z, \eta)$ 具有如下标准形式. 具体地 说, 记 $z=(x, t)$, 不妨假设曲面在 $\left(\mathbf{0}, \boldsymbol{e}_{n}\right)$ 处的法向量 $G(z, \eta)$ 平行于 $t$ 坐标轴, 在不计可忽略因子的 情形下, 相函数 $\phi$ 可以写成如下标准形式. 
引理 $1.1^{[18]}$ 设 $n=2$, 在上述条件下, 有

$$
\phi(x, t, \eta)=\langle x, \eta\rangle+\frac{t}{2} \partial_{t} \partial_{\eta_{1}}^{2} \phi\left(\mathbf{0}, \boldsymbol{e}_{2}\right) \frac{\eta_{1}^{2}}{\eta_{2}}+\eta_{2} \mathcal{E}\left(x, t, \frac{\eta_{1}}{\eta_{2}}\right),
$$

其中 $\eta=\left(\eta_{1}, \eta_{2}\right), \mathcal{E}(x, t, s)$ 满足

$$
\mathcal{E}(x, t, s)=O\left((|x|+|t|)^{2} s^{2}+(|x|+|t|)|s|^{3}\right) .
$$

\section{Euclid 空间半波算子的局部光滑效应}

设 $f$ 是 Schwartz 函数, 波动方程 Cauchy 问题

$$
\left\{\begin{array}{l}
\left(\partial_{t t}-\Delta\right) u=0, \\
u(0, x)=f, \quad \partial_{t} u(0, x)=0
\end{array}\right.
$$

的解 $u$ 可用半波算子 $\mathrm{e}^{\mathrm{i} t \sqrt{-\Delta}}$ 表示成

$$
u(x, t)=\frac{1}{2}\left(\mathrm{e}^{\mathrm{i} t \sqrt{-\Delta}} f+\mathrm{e}^{-\mathrm{i} t \sqrt{-\Delta}} f\right) .
$$

对固定时刻 $t$, Peral ${ }^{[19]}$ 和 Miyachi ${ }^{[20]}$ 证明了下面最优的 $L^{p}$ 估计:

$$
\left\|\mathrm{e}^{\mathrm{i} t \sqrt{-\Delta}} f\right\|_{L^{p}\left(\mathbb{R}^{n}\right)} \leqslant C_{t, p}\|f\|_{L_{s_{p}}^{p}}, \quad s_{p}:=(n-1)\left|\frac{1}{2}-\frac{1}{p}\right|, \quad 1<p<\infty .
$$

由上式估计容易推出下面的时空估计：

$$
\left(\int_{1}^{2}\left\|\mathrm{e}^{\mathrm{i} t \sqrt{-\Delta}} f\right\|_{L^{p}\left(\mathbb{R}^{n}\right)}^{p} d t\right)^{\frac{1}{p}} \lesssim\|f\|_{L_{s p}^{p}\left(\mathbb{R}^{n}\right)} .
$$

相对于 (2.2) 而言, (2.3) 没有从时间平均中赢得任何利润. 一个很自然的问题是, 相对固定时刻的估 计, 能否通过时间平均获得一些正则性? 更准确地说, 是否存在一个 $\varepsilon>0$ 使得

$$
\left(\int_{1}^{2}\left\|\mathrm{e}^{\mathrm{i} t \sqrt{-\Delta}} f(x, t)\right\|_{L^{p}\left(\mathbb{R}^{n}\right)}^{p} d t\right)^{\frac{1}{p}} \lesssim\|f\|_{L_{s_{p}-\varepsilon}^{p}\left(\mathbb{R}^{n}\right)} ?
$$

Sogge ${ }^{[21]}$ 在将 Bourgain 的圆 (circular) 极大函数估计推广到流形测地圆上极大函数估计的过程中, 提 出了下面局部光滑性猜想:

猜想 2.1 (局部光滑性猜想 ${ }^{[21]}$ ) 设 $n \geqslant 2$, 对所有的

$$
\sigma< \begin{cases}\frac{1}{p}, & \text { 若 } \frac{2 n}{n-1}<p<\infty, \\ s_{p}, & \text { 若 } 2<p \leqslant \frac{2 n}{n-1},\end{cases}
$$

成立如下不等式:

$$
\left(\int_{1}^{2}\left\|\mathrm{e}^{\mathrm{i} t \sqrt{-\Delta}} f\right\|_{L^{p}\left(\mathbb{R}^{n}\right)}^{p} d t\right)^{\frac{1}{p}} \lesssim\|f\|_{L_{s_{p}-\sigma}^{p}\left(\mathbb{R}^{n}\right)} .
$$


Sogge ${ }^{[21]}$ 率先获得了 $p>2 、 n=2$ 情形下的部分结果. 随后, Mockenhaupt 等 ${ }^{[22]}$ 通过平方函数 估计方法改进了文献 [21] 的结果. 第一个最佳 (sharp) 正则性结果属于 Wolff [23]. Wolff 引入所谓的 “ $\ell^{p}$ - 分离性不等式” 的概念, 获得了 $n=2 、 p>74$ 时最佳正则性结果. 经历一系列工作 [24-26], 最优 的 “ $\ell^{2}$ - 分离性不等式” 最终被 Bourgain 和 Demeter ${ }^{[27]}$ 证明, 并形成了调和分析中崭新的分离性理 论. 这一理论影响深远, 已经被广泛地应用在偏微分方程、数论和几何测度论等不同数学领域. 例如, Bourgain 等 ${ }^{[28]}$ 对多项式曲线所建立的分离性定理, 一举解决了解析数论中沉睡百年的 Vinogradov 猜 想; 对于局部光滑性估计而言, 作为其直接的推论, 解决了 $p \geqslant \frac{2(n+1)}{n-1} 、 n \geqslant 2$ 对应的局部光滑性估计. 最近, Guth 等 ${ }^{[29]}$ 通过建立最佳平方函数不等式, 完全解决了 $n=2$ 情形下的局部光滑性猜想.

为方便问题转化与归结, 定义区域 $\Gamma \subset \mathbb{R}^{n}$ 如下:

$$
\Gamma:=\left\{\left(\xi^{\prime}, \xi_{n}\right) \in \mathbb{R}^{n} \backslash\{0\}: 1 \leqslant \xi_{n} \leqslant 2,\left|\xi^{\prime}\right| \leqslant \xi_{n}\right\},
$$

对 $\Gamma$ 实施扇形分解. 假设 $R>1$, 在超平面 $x_{n}=1$ 的单位球 $B^{n-1}(0,1) \times\{1\}$ 中, 选取 $R^{-\frac{1}{2}}$ - 极大分 离的点 $\left\{\left(\xi_{\nu}, 1\right)\right\}_{\nu}$. 定义扇形 $\nu$ 如下:

$$
\nu:=\left\{\left(\xi^{\prime}, \xi_{n}\right) \in \mathbb{R}^{n} \backslash\{0\}:\left|\frac{\xi^{\prime}}{\xi_{n}}-\xi_{\nu}\right| \leqslant R^{-\frac{1}{2}}\right\},
$$

设 $\chi_{\nu}$ 为 $\nu$ 的特征函数, 并记 $f_{\nu}=\mathcal{F}^{-1}\left(\hat{f} \chi_{\nu}\right)$.

半波算子的局部光滑性估计的研究方法主要分两种, 其一是建立平板 (slab)- 分解对应的平方函 数估计, 结合 Kakeya 极大函数估计方法; 其二是 Wolff 型的 $\ell^{p}$ - 分离性不等式

$$
\left\|\sum_{\nu} \mathrm{e}^{\mathrm{i} t \sqrt{-\Delta}} f_{\nu}\right\|_{L^{p}\left(\mathbb{R}^{n+1}\right)} \leqslant C_{\varepsilon} R^{\frac{n-1}{2}\left|\frac{1}{2}-\frac{1}{p}\right|+\varepsilon}\left(\sum_{\nu}\left\|\mathrm{e}^{\mathrm{i} t \sqrt{-\Delta}} f_{\nu}\right\|_{L^{p}\left(\mathbb{R}^{n+1}\right)}^{p}\right)^{\frac{1}{p}},
$$

其中 $2 \leqslant p \leqslant \frac{2(n+1)}{n-1}$. 作为 Bourgain-Demeter 的最佳分离性的直接推论, 在不计端点正则性的前提下, 可直接获得 $p \geqslant \frac{2(n+1)}{n-1}$ 情形下的最优局部光滑性估计. 直观上来看, 对于较大的可积指标, $\ell^{p}$ - 分离性 不等式方法比较有效. 但当 $p$ 接近于端点 $\frac{2 n}{n-1}$ 时, 分离性不等式方法的作用已不再有效. 基于这个观 察, 我们可以尝试建立下面更强的平方函数不等式:

$$
\left\|\sum_{\nu} \mathrm{e}^{\mathrm{i} t \sqrt{-\Delta}} f_{\nu}\right\|_{L^{p}\left(\mathbb{R}^{n+1}\right)} \leqslant C_{\varepsilon} R^{\varepsilon}\left\|\left(\sum_{\nu}\left|\mathrm{e}^{\mathrm{i} t \sqrt{-\Delta}} f_{\nu}\right|^{2}\right)^{\frac{1}{2}}\right\|_{L^{p}\left(\mathbb{R}^{n+1}\right)}, \quad 2 \leqslant p \leqslant \frac{2 n}{n-1} .
$$

Guth 等 ${ }^{[29]}$ 建立了 $2+1$ 维空间中几乎最佳平方函数不等式 (2.8). 利用文献 [22] 中的标准方法, 我们 可以得到 $2+1$ 维情形对应的最佳局部光滑性估计. 我们将在附录 A 中给出详细细节, 并列举一些相 关公开问题.

\section{3 局部光滑性估计的应用一举例}

局部光滑性猜想与调和分析中其他核心问题紧密相关, 如 Bochner-Riesz 求和及其衍生的极大 Bochner-Riesz 猜想. Bochner-Riesz 求和源于如何定义 Fourier 逆变换, 即在什么意义下, 成立

$$
\lim _{R \rightarrow \infty}(2 \pi)^{-\frac{n}{2}} \int_{|x| \leqslant R} \hat{f}(\xi) \mathrm{e}^{\mathrm{i} x \xi} d \xi=f(x) ?
$$


容易看出, (3.1) 在分布意义下成立, 但是进一步要问: (3.1) 是否在点态意义下成立? 为了回答这一问 题, 可以引入 $\Phi(\xi)$ - 求和法. 具体而言, 设 $\Phi$ 为快速衰减可积函数, 且 $\Phi(0)=1$. 点态收玫问题就转化 为算子 $S_{t}$ :

$$
S_{t} f(x):=\int_{\mathbb{R}^{n}} \mathrm{e}^{\mathrm{i} x \xi} \Phi\left(\frac{\xi}{t}\right) \hat{f}(\xi) d \xi, \quad t>0
$$

在 $t \rightarrow \infty$ 时的性态. 当 $\Phi$ 是 Gauss 函数时, 对应着 Gauss 求和法. 如果取 $\Phi(\xi)=(1-|\xi|)_{+}^{\delta}, \delta>0$, 则 对应着 Bochner-Riesz 求和法. 考虑 Bochner-Riesz 平均算子 $S_{t}^{\delta}$ :

$$
\int_{\mathbb{R}^{n}} \mathrm{e}^{\mathrm{i} x \xi}\left(1-\left|\frac{\xi}{t}\right|\right)_{+}^{\delta} \hat{f}(\xi) d \xi
$$

记

$$
\delta(p):=\max \left\{n\left|\frac{1}{2}-\frac{1}{p}\right|-\frac{1}{2}, 0\right\} .
$$

猜想 3.1 (极大 Bochner-Riesz 猜想) 设 $n \geqslant 2,2 \leqslant p<\infty, \delta>\delta(p)$, 则

$$
\left(\int_{\mathbb{R}^{n}} \sup _{t>0}\left|S_{t}^{\delta} f(x)\right|^{p} d x\right)^{\frac{1}{p}} \lesssim\|f\|_{L^{p}\left(\mathbb{R}^{n}\right)} .
$$

如果 (3.4) 成立, 采用常规方法不难推出, 对满足上述条件的 $\delta 、 n$ 和 $p$, 有

$$
S_{t}^{\delta} f \rightarrow f \text { 几乎处处成立, } \forall f \in L^{p}\left(\mathbb{R}^{n}\right) \text {. }
$$

下面来说明局部光滑性猜想 2.1 意味着极大 Bochner-Riesz 猜想.

命题 3.1 局部光滑性猜想意味着极大 Bochner-Riesz 猜想.

我们可选择合适的 $\psi \in C_{c}^{\infty}\left(\mathbb{R}^{n}\right)$, 把 $(1-|\xi|)_{+}^{\delta}$ 分解成

$$
(1-|\xi|)_{+}^{\delta}=r(|\xi|)+\sum_{k=1}^{\infty} 2^{-k \delta} \psi\left(2^{k}(1-|\xi|)\right),
$$

其中 $r(|\xi|) \in C_{c}^{\infty}\left(\mathbb{R}^{n}\right)$. 注意到

$$
\left|\int_{\mathbb{R}^{n}} \mathrm{e}^{\mathrm{i} x \xi} r(|\xi|) \hat{f}(\xi) d \xi\right| \lesssim M f(x),
$$

其中 $\boldsymbol{M}$ 表示 Hardy-Littlewood 极大函数. 注意到

$$
\|M f\|_{L^{p}} \lesssim\|f\|_{L^{p}}, \quad p \geqslant 2,
$$

只需要估计剩余项. 由 Minkowski 不等式

$$
\begin{aligned}
& \left(\int_{\mathbb{R}^{n}} \sup _{t>0}\left|\int \mathrm{e}^{\mathrm{i} x \xi} \sum_{k=1}^{\infty} 2^{-k \delta} \psi\left(2^{k}(1-|t \xi|)\right) \hat{f}(\xi) d \xi\right|^{p} d x\right)^{\frac{1}{p}} \\
& \quad \lesssim \sum_{k=1}^{\infty} 2^{-k \delta}\left(\int_{\mathbb{R}^{n}} \sup _{t>0}\left|\int \mathrm{e}^{\mathrm{i} x \xi} \psi\left(2^{k}(1-|t \xi|)\right) \hat{f}(\xi) d \xi\right|^{p} d x\right)^{\frac{1}{p}},
\end{aligned}
$$

从而问题归结为证明对任意 $\varepsilon>0$, 有

$$
\left(\int_{\mathbb{R}^{n}} \sup _{t>0}\left|\int \mathrm{e}^{\mathrm{i} x \xi} \psi\left(2^{k}(1-|t \xi|)\right) \hat{f}(\xi) d \xi\right|^{p} d x\right)^{\frac{1}{p}} \lesssim 2^{k(\delta(p)+\varepsilon)}\|f\|_{L^{p}\left(\mathbb{R}^{n}\right)} .
$$


记

$$
A_{t}^{\lambda} f(x):=\int \mathrm{e}^{\mathrm{i} x \xi} \psi(\lambda-|t \xi|) \hat{f}(\xi) d \xi
$$

令 $\lambda \gg 1,(3.5)$ 等价于证明

$$
\left\|\sup _{t>0}\left|A_{t}^{\lambda} f\right|\right\|_{L^{p}\left(\mathbb{R}^{n}\right)} \lesssim \lambda^{\delta(p)+\varepsilon}\|f\|_{L^{p}\left(\mathbb{R}^{n}\right)} .
$$

由 Littlewood-Paley 定理和正交性, 仅需证明

$$
\left\|\sup _{t \in(1,2)}\left|A_{t}^{\lambda} f\right|\right\|_{L^{p}\left(\mathbb{R}^{n}\right)} \lesssim \lambda^{\delta(p)+\varepsilon}\|f\|_{L^{p}\left(\mathbb{R}^{n}\right)}, \quad \operatorname{supp} \hat{f}(\xi) \subset\left(\frac{\lambda}{2}, \lambda\right) .
$$

显然, (3.7) 可从下面的估计得到:

$$
\left\|A_{t(x)}^{\lambda} f\right\|_{L^{p}\left(\mathbb{R}^{n}\right)} \lesssim \lambda^{\sigma(p)+\varepsilon}\|f\|_{L^{p}\left(\mathbb{R}^{n}\right)},
$$

其中 $t(x) \in(1,2)$ 为可测函数.

为方便使用局部光滑性估计, 实施如下转换:

$$
\begin{aligned}
\psi(\lambda-|t(x) \xi|) & =\int_{\mathbb{R}} \check{\psi}(s) \mathrm{e}^{-\mathrm{i} \lambda s} \mathrm{e}^{\mathrm{i} s t(x)|\xi|} d s \\
& =t(x)^{-1} \int_{\mathbb{R}} \check{\psi}\left(\frac{s}{t(x)}\right) \mathrm{e}^{-\mathrm{i} \lambda s / t(x)} \mathrm{e}^{\mathrm{i} s|\xi|} d s .
\end{aligned}
$$

将 (3.9) 代入到 (3.7), 利用局部光滑性估计 $(2.6)$, 注意到 $1 \leqslant t(x) \leqslant 2$ 及 $\check{\psi}$ 的快速衰减性质, 容易推 出 (3.8). 这说明局部光滑性猜想 2.1 意味着极大 Bochner-Riesz 猜想.

极大 Bochner-Riesz 猜想与下面几个著名的猜想紧密相关:

猜想 $3.2^{[5]}$ (Bochner-Riesz 猜想) 设 $1 \leqslant p \leqslant \infty$. 如果 $\delta>\delta(p)$, 则

$$
\left\|S_{1}^{\delta} f\right\|_{L^{p}\left(\mathbb{R}^{n}\right)} \lesssim\|f\|_{L^{p}\left(\mathbb{R}^{n}\right)}
$$

猜想 $\mathbf{3 . 3}{ }^{[5]}$ (限制性猜想) 设 $n \geqslant 2,(p, q)$ 满足

$$
\left\{(p, q): \frac{1}{q}=\frac{n+1}{n-1} \frac{1}{p^{\prime}}, 1 \leqslant p<\frac{2 n}{n-1}\right\},
$$

则

$$
\left\|\left.\hat{f}\right|_{S}\right\|_{L^{q}(S, d \sigma)} \leqslant C\|f\|_{L^{p}\left(\mathbb{R}^{n}\right)}, \quad \forall f \in L^{p}\left(\mathbb{R}^{n}\right) .
$$

猜想 3.4 (Kakeya 极大函数猜想 (离散版本) ) 设 $\Omega \subset S^{n-1}$ 为一组极大 $\delta^{1 / 2}$ 分离的点集构成的 集合, 设 $\left\{T_{\omega}\right\}_{\omega \in \Omega}$ 为一列尺度为 $\delta^{-1} \times \delta^{-1 / 2} \times \cdots \times \delta^{-1 / 2}$ 的长方体的集合, 其中 $T_{\omega}$ 的方向为 $\omega$, 则

$$
\left\|\sum_{\omega \in \Omega} \chi_{T_{\omega}}\right\|_{L^{\frac{n}{n-1}\left(\mathbb{R}^{n}\right)}} \lesssim\left(\sum_{\omega \in \Omega}\left|T_{\omega}\right|\right)^{\frac{n-1}{n}} .
$$

猜想 3.5 (Kakeya 猜想) 称集合 $E \subset \mathbb{R}^{n}$ 为 Kakeya 集, 如果 $E$ 中包含有任意方向的单位线段, 则任一 Kakeya 集的 Hausdorff 维数是 $n$. 
注 3.1 上述几个猜想之间有如下联系:

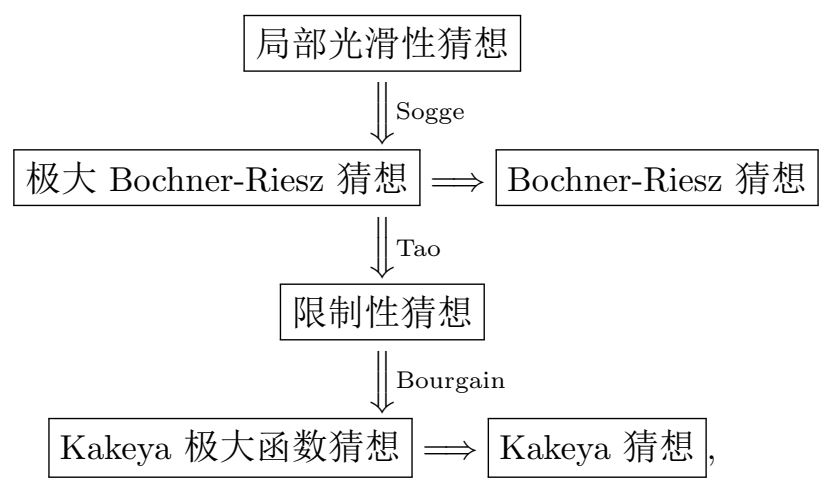

更多的讨论可参见文献 [30-37].

\section{4 基本工具}

为了完整起见, 接下来不加证明地给出 Whitney 分解引理, 详细的讨论可参见文献 $[18,38,39]$.

引理 4.1 (Whitney 分解引理) 设 $\xi, \eta \in \mathbb{R}^{n}$, 则存在唯一的二进制正方体对 $\left(Q_{1}, Q_{2}\right) \subset \mathbb{R}^{n}$ 满足

(i) $\xi \in Q_{1}, \eta \in Q_{2}$;

(ii) $\ell\left(Q_{1}\right)=\ell\left(Q_{2}\right)$;

(iii) $\ell\left(Q_{1}\right) \leqslant \operatorname{dist}\left(Q_{1}, Q_{2}\right) \leqslant 4 \sqrt{n} \ell\left(Q_{1}\right)$,

其中 $\ell\left(Q_{1}\right)$ 和 $\ell\left(Q_{2}\right)$ 分别表示正方体 $Q_{1}$ 和 $Q_{2}$ 的边长.

总假设 $\left(\mathrm{H}_{1}\right)$ 和 $\left(\mathrm{H}_{2}\right)$ 的定量版本: 对给定充分大的 $N>1$, 相函数的高阶导数满足一致估计

$$
\left|\partial_{\eta}^{\beta} \partial_{z}^{\alpha} \phi(z, \eta)\right| \leqslant \frac{1}{2}, \quad 0 \leqslant|\beta| \leqslant N, \quad|\alpha|=2 \text {, 对所有的 }(z, \eta) \in \operatorname{supp} a .
$$

否则选取 $A \gg 1$, 用新的相函数 $A \phi(z / A, \eta)$ 去替换 $\phi(z, \eta)$, 则对新的相函数总有 (4.1) 成立. 详细讨 论可参见文献 $[18,40-42]$.

设 $\Upsilon_{x, t}: \eta \rightarrow \partial_{x} \phi(x, t, \eta)$. 选取 $\varepsilon_{0}$ 充分小, 使得映射 $\Upsilon_{x, t}$ 在 $B\left(\boldsymbol{e}_{2}, \varepsilon_{0}\right)$ 上是微分同胚. 若用 $\Psi_{x, t}(\xi)=\Upsilon_{x, t}^{-1}(\xi)$ 表示 $\Upsilon_{x, t}$ 的逆映射, 容易看出

$$
\partial_{x} \phi\left(x, t, \Psi_{x, t}(\xi)\right)=\xi .
$$

对 (4.2) 两边关于 $\xi$ 求导, 直接推知

$$
\left[\partial_{x, \eta}^{2} \phi\right]\left(x, t, \Psi_{x, t}(\xi)\right) \partial_{\xi} \Psi_{x, t}(\xi)=\mathrm{Id} .
$$

这表明

$$
\operatorname{det} \partial_{\xi} \Psi_{x, t}(\xi) \neq 0, \quad \forall(x, t) \in B\left(\mathbf{0}, \varepsilon_{0}\right), \quad \forall \xi \in \Upsilon_{x, t}\left(B\left(\boldsymbol{e}_{2}, \varepsilon_{0}\right)\right)
$$

设

$$
q(x, t, \xi)=\partial_{t} \phi\left(x, t, \Psi_{x, t}(\xi)\right)
$$

则

$$
\partial_{t} \phi(x, t, \eta)=q\left(x, t, \partial_{x} \phi(x, t, \eta)\right) .
$$


设 $j=1,2$, 考虑如下两个振荡积分算子:

$$
W_{\lambda}^{j} f(z)=\int \mathrm{e}^{\mathrm{i} \lambda \phi_{j}(z, \eta)} a_{j}(z, \eta) f(\eta) d \eta, \quad z=(x, t) \in \mathbb{R}^{2} \times \mathbb{R},
$$

其中 $\phi_{j}$ 满足 $\left(\mathrm{H}_{1}\right)$ 和 $\left(\mathrm{H}_{2}\right)$ 以及 $a_{j} \in C_{c}^{\infty}\left(\mathbb{R}^{3} \times \mathbb{R}^{2}\right)$.

我们拟采用下面双线性振荡积分估计建立平方函数估计, 双线性振荡估计是 Wolff [43] 双线性限 制性定理在变系数情形下的自然推广. 特别地, 对于每一个多重指标 $\beta \in \mathbb{N}^{3}$, 假设

$$
\text { 对于所有的 }(z, \eta) \in \operatorname{supp} a, \quad\left|\partial_{z}^{\beta} \phi_{j}(z, \eta)\right| \leqslant A_{\beta}, \quad 0 \leqslant|\beta| \leqslant N, \quad j=1,2,
$$

我们有下面的振荡积分估计:

定理 $4.1^{[18]}$ 设 $j=1,2, \phi_{j}$ 为关于变量 $\eta$ 的 1- 次齐次光滑函数, 且满足条件 $\left(\mathrm{H}_{1}\right)$ 和 $\left(\mathrm{H}_{2}\right)$. 假 设 $a_{j}$ 的支集充分小, 使得在 $\operatorname{supp} a_{j}$ 上 $\phi_{j}$ 满足 (4.6), $\partial_{x} \phi_{j} \neq 0$, 以及

$$
\operatorname{rank} \partial_{\eta \eta}^{2} q_{j}\left(x, t, \partial_{x} \phi_{j}\left(x, t, \eta^{(j)}\right)\right)=1 .
$$

此外, 对 $\left(z, \eta^{(1)}\right) \in \operatorname{supp} a_{1}$ 和 $\left(z, \eta^{(2)}\right) \in \operatorname{supp} a_{2}$, 下面条件成立:

$$
\left|\left\langle\frac{\partial_{x} \phi_{j}\left(z, \eta^{(j)}\right)}{\left|\partial_{x} \phi_{j}\left(z, \eta^{(j)}\right)\right|}, \partial_{\eta} q_{1}\left(z, \partial_{x} \phi_{1}\left(z, \eta^{(1)}\right)\right)-\partial_{\eta} q_{2}\left(z, \partial_{x} \phi_{2}\left(z, \eta^{(2)}\right)\right)\right\rangle\right| \geqslant c_{0}>0,
$$

则对任意的 $p \geqslant \frac{5}{3}$, 总成立下面双线性振荡积分估计:

$$
\left\|W_{\lambda}^{1} f W_{\lambda}^{2} g\right\|_{L^{p}\left(\mathbb{R}^{2+1}\right)} \leqslant C\left(A_{\beta}, \varepsilon, c_{0}\right) \lambda^{-\frac{3}{p}+\varepsilon}\|f\|_{L^{2}\left(\mathbb{R}^{2}\right)}\|g\|_{L^{2}\left(\mathbb{R}^{2}\right)},
$$

其中隐性常数只涉及有限的多重指标 $\beta$.

\section{5 双线性方法}

当 $n=2$ 时, 利用 Beltran 等 ${ }^{[41]}$ 建立的最佳型 $L^{6}$ - 估计与平凡的 $L^{2}$ - 估计插值, 只能重新获得文 献 [22] 中的 $L^{4}-L^{4}$ 相应结果. 然而, 通过双线性方法, 可以改进文献 [22] 中对应的局部光滑性估计.

定理 $5.1^{[44]}$ 设 $Z$ 和 $Y$ 是分别满足 $\operatorname{dim} Z=3$ 和 $\operatorname{dim} Y=2$ 的光滑紧 Riemann 流形. 假设 $\mathscr{F} \in I^{\sigma-\frac{1}{4}}(Z, Y ; \mathscr{C})$, 典则关系 $\mathscr{C}$ 满足电影型曲率条件, 则估计

$$
\|\mathscr{F} f\|_{L_{\mathrm{loc}}^{p}(Z)} \leqslant C\|f\|_{L_{\mathrm{comp}}^{p}(Y)}, \quad \sigma<-\sigma(p)
$$

成立, 其中 $2 \leqslant p \leqslant 6$,

$$
\sigma(p)= \begin{cases}\frac{1}{8}-\frac{1}{4 p}, & 2 \leqslant p \leqslant \frac{10}{3} \\ \frac{5}{16}-\frac{7}{8 p}, & \frac{10}{3} \leqslant p \leqslant 6\end{cases}
$$

注 5.1 (i) 对 $\mathscr{F} \in I^{\sigma-\frac{1}{4}}(Z, Y ; \mathscr{C})$ 而言, 以 $L^{4}-L^{4}$ 估计为标准仅需要 $\sigma<-\frac{3}{32}$. 这与文献 [22] 中 的结果相比较, 正则性指标提升了 $\frac{1}{32}$;

(ii) 该问题也可采用多线性方法, 适当提升正则性指标, 详见文献 [45-47]. 
我们沿用 Mockenhaupt 等 ${ }^{[22]}$ 的策略, 证明定理 5.1 的关键因素之一是变系数 Kakeya 极大函数 估计, 这种估计曾出现在 Córdoba ${ }^{[48]}$ 关于 Bochner-Riesz 乘子定理的研究工作中.

设 $(y, \xi) \in \Pi_{T^{*} Y}(\mathscr{C})$, 令

$$
\gamma_{y, \xi}=\{z \in Z:(z, \zeta, y, \xi) \in \mathscr{C}, \text { 对于某些 } \zeta\}
$$

是嵌入到 $Z$ 中的光滑曲线. 取定 $Z$ 上的光滑度量并选择 $\delta>0$ 充分小, 定义

$$
R_{y, \xi}^{\delta}:=\left\{z \in Z: \operatorname{dist}\left(z, \gamma_{y, \xi}\right)<\delta\right\}
$$

设 $\alpha \in C_{0}^{\infty}(Y \times Z)$, 令

$$
\mathcal{M}_{\delta} g(y)=\sup _{\xi \in \Pi_{T_{y}^{*} Y}(\mathscr{C})} \frac{1}{\operatorname{Vol}\left(R_{y, \xi}^{\delta}\right)}\left|\int_{R_{y, \xi}^{\delta}} \alpha(y, z) g(z) d z\right|
$$

利用下面定理 5.2 和平凡的 $L^{\infty} \rightarrow L^{\infty}$ 估计进行插值, 容易推出

$$
\left\|\mathcal{M}_{\delta}\right\|_{L^{p} \rightarrow L^{p}} \leqslant C\left(\log \frac{1}{\delta}\right)^{\frac{1}{2}}, \quad \forall 2 \leqslant p \leqslant \infty .
$$

定理 $5.2^{[22]}$ 假设 $Z$ 和 $Y$ 分别是维数为 3 和 2 的准紧光滑流形并被赋予光滑度量. 假设典则 关系 $\mathscr{C}$ 满足电影型曲率条件, 则存在常数 $C$ 使得

$$
\left\|\mathcal{M}_{\delta} g\right\|_{L^{2}(Y)} \leqslant C \sqrt{\log \frac{1}{\delta}}\|g\|_{L^{2}(Z)}, \quad \forall g \in L^{2}(Z) .
$$

类似于 Euclid 情形, 借助于变系数极大 Kakeya 函数估计 (5.5)、Littlewood-Paley 分解、尺度伸 缩 (rescaling) 技术与角分解, 可把 (5.1) 的证明归结成如下的平方函数估计:

$$
\left\|T_{\lambda} f\right\|_{L^{p}\left(\mathbb{R}^{2+1}\right)} \lesssim \lambda^{-\sigma(p)}\left\|\left(\sum_{\nu}\left|T_{\lambda}^{\nu} f\right|^{2}\right)^{\frac{1}{2}}\right\|_{L^{p}\left(\mathbb{R}^{2+1}\right)}+\operatorname{RapDec}(\lambda)\|f\|_{L^{p}\left(\mathbb{R}^{2}\right)},
$$

其中

$$
\left\{\begin{array}{l}
T_{\lambda}(f)(z):=\int_{\mathbb{R}^{2}} \mathrm{e}^{\mathrm{i} \lambda \phi(z, \eta)} a(z, \eta) f(\eta) d \eta, \quad \forall \lambda \gg 1, \\
T_{\lambda} f(z)=\sum_{\nu} T_{\lambda}^{\nu} f(z), \quad T_{\lambda}^{\nu} f(z)=\int_{\mathbb{R}^{2}} \mathrm{e}^{\mathrm{i} \lambda \phi(z, \eta)} a^{\nu}(z, \eta) f(\eta) d \eta \\
a^{\nu}(z, \eta):=\chi_{\nu}(\eta) a(z, \eta), \quad a(z, \eta) \in C_{0}^{\infty}\left(\mathbb{R}^{2+1} \times \mathbb{R}^{2}\right),
\end{array}\right.
$$

$\left\{\chi_{\nu}(\eta)\right\}$ 是单位圆上特征函数对应的单位分解在 $\mathbb{R}^{2} \backslash\{0\}$ 上的零齐次扩张, 满足

$$
\left\{\begin{array}{l}
\sum_{0 \leqslant \nu \leqslant N_{\lambda}} \chi_{\nu}(\eta) \equiv 1, \quad \forall \eta \in \mathbb{R}^{2} \backslash\{0\} \\
\left|\partial^{\alpha} \chi_{\nu}(\eta)\right| \leqslant C_{\alpha} \lambda^{\frac{|\alpha|}{2}}, \quad \forall \alpha \in \mathbb{N}^{2}, \quad|\eta|=1 .
\end{array}\right.
$$

根据振荡积分的稳定性定理, 我们可不妨假设振幅函数 $a$ 是变量可分离的, 即

$$
a(z, \eta)=a_{1}(z) a_{2}(\eta)=a_{1}(x, t) a_{2}(\eta)
$$


其中 $a_{1} \in C_{c}^{\infty}\left(B\left(0, \varepsilon_{0}\right)\right), a_{2} \in C_{c}^{\infty}\left(B\left(\boldsymbol{e}_{2}, \varepsilon_{0}\right)\right)$.

对一般情形, 总可以通过如下变换:

$$
T_{\lambda} f(z)=\int_{\mathbb{R}^{3}} \mathrm{e}^{\mathrm{i}(z, \xi)}\left(\int_{\mathbb{R}^{2}} \mathrm{e}^{\mathrm{i} \lambda \phi(z, \eta)} \psi(z) \widehat{a}(\xi, \eta) f(\eta) d \eta\right) d \xi
$$

归结成变量可分离情形. 容易看出 $\xi \mapsto \widehat{a}(\xi, \eta)$ 是 Schwartz 函数, 其中 $\psi(z)$ 是一个具有紧支撑的光滑 函数且在 $\operatorname{supp}_{z} a$ 上恒等于 1 .

利用 Lee ${ }^{[18]}$ 建立的双线性振荡积分估计可提升文献 [22] 中的平方函数估计, 从而进一步改进满 足电影型曲率条件的 Fourier 积分算子的局部光滑性估计. 实现该目标涉及两个重要步骤, 其一是采 用 Whitney 分解引理和抛物尺度变换技术, 把问题归结成证明与之等价的双线性平方函数估计; 其二, 如何将双线性平方函数估计转化成双线性振荡积分估计, 这需要利用局部常数性质、正交性和扰动性 引理来实现这个目的.

\section{1 双线性等价形式}

根据上面的讨论, (5.1) 的证明归结为证明 (5.6) 在 $p=\frac{10}{3}$ 情形下对应的特例. 为此我们采用文 献 $[25,39]$ 中发展的双线性方法与扰动方法. 扰动方法的理念意味着在物理空间尺度充分小的意义下, 振荡积分算子可以被相应的延拓算子逼近.

我们的思路是将 (5.6) 转化成等价的双线性形式, 以方便地使用双线性振荡积分估计. 利用 Hölder 不等式, 线性估计 (5.6) 意味着双线性估计. 然而反方向的蕴涵关系需要细致的分析.

命题 5.1 假设 $\Omega$ 和 $\Omega^{\prime}$ 是分别包含 $\nu$ 和 $\nu^{\prime}$ 的两个方向集, 满足如下角向分离条件:

$$
\operatorname{Ang}\left(\theta_{\nu}, \theta_{\nu^{\prime}}\right) \approx_{\varepsilon_{0}} 1, \quad \forall\left(\nu, \nu^{\prime}\right) \in \Omega \times \Omega^{\prime},
$$

其中 $\operatorname{Ang}\left(\theta_{\nu}, \theta_{\nu^{\prime}}\right)$ 刻画扇形 $\theta_{\nu}$ 与 $\theta_{\nu^{\prime}}$ 之间的夹角. 设 $T_{\lambda}^{\nu}$ 是 (5.7) 中定义的算子, 其中相函数 $\phi$ 满足 条件 $\left(\mathrm{H}_{1}\right) 、\left(\mathrm{H}_{2}\right)$ 和 $a \in C_{c}^{\infty}\left(\mathbb{R}^{3} \times \mathbb{R}^{2}\right)$. 如果对于任意的函数 $g$ 和 $h$, 在不计余项 $\operatorname{RapDec}(\lambda)$ 的情形下,

$$
\begin{aligned}
& \left\|\sum_{\nu \in \Omega} T_{\lambda}^{\nu} g \sum_{\nu^{\prime} \in \Omega^{\prime}} T_{\lambda}^{\nu^{\prime} h}\right\|_{L^{\frac{5}{3}}\left(\mathbb{R}^{2+1}\right)} \\
& \quad \lesssim \lambda^{1 / 10}\left\|\left(\sum_{\nu \in \Omega}\left|T_{\lambda}^{\nu} g\right|^{2}\right)^{\frac{1}{2}}\right\|_{L^{\frac{10}{3}\left(\mathbb{R}^{2+1}\right)}}\left\|\left(\sum_{\nu^{\prime} \in \Omega}\left|T_{\lambda}^{\nu^{\prime}} h\right|^{2}\right)^{\frac{1}{2}}\right\|_{L^{\frac{10}{3}\left(\mathbb{R}^{2+1}\right)}},
\end{aligned}
$$

其中隐性常数依赖于 (4.10) 中的常数 $c_{0}$ 和有限 $\beta$ 的常数 $A_{\beta}$, 则有 (5.6), 其中 $p=\frac{10}{3}$.

证明 首先在二进制框架下, 对两扇形区域的笛卡尔积所构成的集合实施非齐次 Whitney 分解, 使得二进尺度落在 $\left(\lambda^{-1 / 2}, 1\right)$ 上. 其次分离出远离对角线且落在相同二进尺度中的双线性项. 为了利 用引理中的假设, 最后采用抛物尺度伸缩技术把落在相同尺度中双线性项化成引理 5.1 中出现的标准 情形.

\section{第 1 步 正交性技术}

设 $j_{0}$ 是使得 $2^{j 0} \leqslant \lambda^{1 / 2}$ 成立的最大整数. 对任意的 $j \in \mathbb{Z}$ 满足 $\left|\log _{2} \varepsilon_{0}\right| \leqslant j \leqslant j_{0}$, 用 $\left\{\theta_{j, \ell}\right\}_{\ell}$ 表 示尺度为 $\approx 2^{-j}$ 的扇形构成的集合, 其中 $\ell \in\left\{1,2, \ldots, \ell_{j}\right\}$. 设 $\ell_{j} \approx 2^{j}$, 对任意的 $\ell$, 记 $\theta_{j, \ell}$ 是包含 $\approx 2^{-j} \lambda^{1 / 2}$ 个连续扇形 $\theta_{\nu}$ 的集合.

对集合 $\mathcal{C}\left(\boldsymbol{e}_{2}, \varepsilon_{0}\right) \times \mathcal{C}\left(\boldsymbol{e}_{2}, \varepsilon_{0}\right)$ 做如下 Whitney 分解:

$$
\mathcal{C}\left(\boldsymbol{e}_{2}, \varepsilon_{0}\right) \times \mathcal{C}\left(\boldsymbol{e}_{2}, \varepsilon_{0}\right)
$$




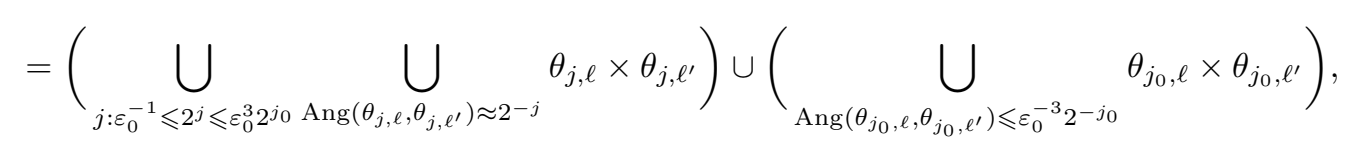

其中 $\operatorname{Ang}\left(\theta_{j, \ell}, \theta_{j, \ell^{\prime}}\right) \approx 2^{-j}$ 表示 $2^{-(j+1)} \leqslant \operatorname{Ang}\left(\theta_{j, \ell}, \theta_{j, \ell^{\prime}}\right) \leqslant 2^{-(j-1)}$.

对 $1 \leqslant \ell \leqslant \ell_{j}$, 令

$$
\chi_{j, \ell}(\eta)=\sum_{\nu: \theta_{\nu} \subset \theta_{j, \ell}} \chi_{\nu}(\eta), \quad a^{j, \ell}(x, t, \eta)=\chi_{j, \ell}(\eta) a(x, t, \eta) .
$$

定义

$$
\left(T_{\lambda}^{j, \ell} f\right)(x, t)=\int \mathrm{e}^{\mathrm{i} \lambda \phi(x, t, \eta)} a^{j, \ell}(x, t, \eta) f(\eta) d \eta
$$

简单计算有

$$
\begin{aligned}
\left(\left(T_{\lambda} f\right)(x, t)\right)^{2}= & \sum_{\left(\ell, \ell^{\prime}\right) \in \Lambda_{j_{0}}}\left(T_{\lambda}^{j_{0}, \ell} f\right)(x, t)\left(T_{\lambda}^{j_{0}, \ell^{\prime}} f\right)(x, t) \\
& +\sum_{j: \varepsilon_{0}^{-1} \leqslant 2^{j} \leqslant \varepsilon_{0}^{3} j^{j_{0}}} \sum_{\left(\ell, \ell^{\prime}\right) \in \Lambda_{j}}\left(T_{\lambda}^{j, \ell} f\right)(x, t)\left(T_{\lambda}^{j, \ell^{\prime}} f\right)(x, t),
\end{aligned}
$$

其中 $\Lambda_{j}$ 表示所有满足条件 $\operatorname{Ang}\left(\theta_{j, \ell}, \theta_{j, \ell^{\prime}}\right) \approx 2^{-j}, \varepsilon_{0}^{-1} \leqslant 2^{j} \leqslant \varepsilon_{0}^{3} 2^{j_{0}}$ 的指标 $\left(\ell, \ell^{\prime}\right)$ 的集合, $\Lambda_{j_{0}}$ 表示所有 满足条件 $\operatorname{Ang}\left(\theta_{j_{0}, \ell}, \theta_{j_{0}, \ell^{\prime}}\right) \leqslant \varepsilon_{0}^{-3} 2^{-j_{0}}$ 的指标 $\left(\ell, \ell^{\prime}\right)$ 的集合. 由 Minkowski 不等式得

$$
\begin{aligned}
\left\|T_{\lambda} f\right\|_{L_{x, t}^{\frac{10}{3}}}^{2} & =\left\|\left(T_{\lambda} f\right)^{2}\right\|_{L_{x, t}^{\frac{5}{3}}} \\
\leqslant & \left\|\sum_{\left(\ell, \ell^{\prime}\right) \in \Lambda_{j_{0}}}\left(T_{\lambda}^{j_{0}, \ell} f\right)\left(T_{\lambda}^{j_{0}, \ell^{\prime}} f\right)\right\|_{L_{x, t}^{\frac{5}{3}}}\left\|\sum_{j: \varepsilon_{0}^{-1} \leqslant 2^{j} \leqslant \varepsilon_{0}^{3} 2^{j 0}}\right\| \sum_{\left(\ell, \ell^{\prime}\right) \in \Lambda_{j}}\left(T_{\lambda}^{j, \ell} f\right)\left(T_{\lambda}^{j, \ell^{\prime}} f\right) \|_{L_{x, t}^{\frac{5}{3}}} .
\end{aligned}
$$

对 (5.12) 不等式右端第一项而言, 注意到每个 $\theta_{j_{0}, \ell}$ 仅包含有限多 $\theta_{\nu}$, 根据 Schur 准则, 有

$$
\left\|\sum_{\left(\ell, \ell^{\prime}\right) \in \Lambda_{j_{0}}}\left(T_{\lambda}^{j_{0}, \ell} f\right)\left(T_{\lambda}^{j_{0}, \ell^{\prime}} f\right)\right\|_{L^{\frac{5}{3}}\left(\mathbb{R}_{x, t}^{2+1}\right)} \lesssim\left\|\left(\sum_{\nu}\left|T_{\lambda}^{\nu}(f)\right|^{2}\right)^{\frac{1}{2}}\right\|_{L^{\frac{10}{3}}\left(\mathbb{R}_{x, t}^{2+1}\right)}^{2} .
$$

下面利用条件 (5.9) 实施 (5.12) 的估计. 在不计余项 $\operatorname{Rap} \operatorname{Dec}(\lambda)$ 的意义下, 通过充分开发正交性 质证明不等式

$$
\left\|\sum_{\left(\ell, \ell^{\prime}\right) \in \Lambda_{j}}\left(T_{\lambda}^{j, \ell} f\right)\left(T_{\lambda}^{j, \ell^{\prime}} f\right)\right\|_{L^{\frac{5}{3}}\left(\mathbb{R}_{x, t}^{2+1}\right)} \lesssim\left(\sum_{\left(\ell, \ell^{\prime}\right) \in \Lambda_{j}}\left\|\left(T_{\lambda}^{j, \ell} f\right)\left(T_{\lambda}^{j, \ell^{\prime}} f\right)\right\|_{L^{\frac{5}{3}}\left(\mathbb{R}_{x, t}^{2+1}\right)}^{\frac{5}{3}}\right)^{\frac{3}{5}}
$$

成立.

下面采用 Lee ${ }^{[18]}$ 的方法证明. 设 $\psi(x)$ 是支撑在 $B\left(0, \varepsilon_{0}\right)$ 上的光滑函数, 令 $\psi_{j}^{\mu}(x):=\psi\left(2^{j} x-\mu\right)$, 则有

$$
\sum_{\mu \in \varepsilon_{0} \mathbb{Z}^{2}} \psi_{j}^{\mu}(x) \equiv 1
$$


令 $\Phi\left(x, t, \eta, \eta^{\prime}\right)=\phi(x, t, \eta)+\phi\left(x, t, \eta^{\prime}\right)$, 定义

$$
\begin{aligned}
& \mathcal{P}_{\lambda, \ell, \ell^{\prime}}^{\mu, j}\left(\xi, t, \eta, \eta^{\prime}\right)=\int \mathrm{e}^{\mathrm{i} \lambda \Phi\left(x, t, \eta, \eta^{\prime}\right)-\mathrm{i} x \cdot \xi} A_{\ell, \ell^{\prime}}^{\lambda, j}\left(x, t, \eta, \eta^{\prime}\right) \psi_{j}^{\mu}(x) d x, \\
& \mathcal{A}_{\lambda, \ell, \ell^{\prime}}^{\mu, j}(f)(x, t)=\psi_{j}^{\mu}(x) \iint \mathrm{e}^{\mathrm{i} \lambda \Phi\left(x, t, \eta, \eta^{\prime}\right)} A_{\ell, \ell^{\prime}}^{\lambda, j}\left(x, t, \eta, \eta^{\prime}\right) f(\eta) f\left(\eta^{\prime}\right) d \eta d \eta^{\prime},
\end{aligned}
$$

其中

$$
A_{\ell, \ell^{\prime}}^{\lambda, j}\left(x, t, \eta, \eta^{\prime}\right)=a_{\lambda}^{j, \ell}(x, t, \eta) a_{\lambda}^{j, \ell^{\prime}}\left(x, t, \eta^{\prime}\right) .
$$

记 $r=|\eta|, r^{\prime}=\left|\eta^{\prime}\right|$, 用 $\kappa_{j}^{\ell}$ 和 $\kappa_{j}^{\ell^{\prime}}$ 分别表示 $\theta_{j, \ell}$ 和 $\theta_{j, \ell^{\prime}}$ 的中心. 注意到 $\psi_{j}^{\mu}(x)$ 的支集性质及雉条件, 有

$$
\partial_{x}\left(\lambda \Phi\left(x, t, \eta, \eta^{\prime}\right)-x \xi\right)=\lambda \partial_{x} \Phi\left(2^{-j} \mu, t, r \kappa_{j}^{\ell}, r^{\prime} \kappa_{j}^{\ell^{\prime}}\right)-\xi+O\left(\lambda 2^{-j}\right) .
$$

对 $\left(\ell, \ell^{\prime}\right) \in \Lambda_{j}, \angle\left(\eta, \kappa_{j}^{\ell}\right) \leqslant \varepsilon_{0} 2^{-j}$ 和 $\angle\left(\eta^{\prime}, \kappa_{j}^{\ell^{\prime}}\right) \leqslant \varepsilon_{0} 2^{-j}$, 由分部积分推出映射 $\xi \rightarrow \mathcal{P}_{\lambda, \ell, \ell^{\prime}}^{\mu, j}\left(\xi, t, \eta, \eta^{\prime}\right)$ 在长 方形

$$
\mathcal{R}_{\lambda, \ell}^{\mu, j}(t):=\left\{\xi \in \mathbb{R}^{2}:\left|\xi-\lambda r \partial_{x} \phi\left(2^{-j} \mu, t, \kappa_{j}^{\ell}\right)\right| \lesssim \lambda 2^{-j}, r \in\left(2-2 \varepsilon_{0}, 2+2 \varepsilon_{0}\right)\right\}
$$

之外快速衰减. 由 $\lambda^{\varepsilon} \mathcal{R}_{\lambda, \ell}^{\mu, j}(t)$ 可以诱导定义一个光滑的 bump 函数 $R_{\lambda, \ell}^{\mu, j}(\xi, t)$, 从而有

$$
\left\|\left(\operatorname{Id}-R_{\lambda, \ell}^{\mu, j}\left(D_{x}, t\right)\right) \mathcal{A}_{\lambda, \ell, \ell^{\prime}}^{\mu, j}(f)(t, \cdot)\right\|_{L^{10 / 3}\left(\mathbb{R}_{x}^{2}\right)} \lesssim \operatorname{RapDec}(\lambda)\|f\|_{L^{10 / 3}\left(\mathbb{R}^{2}\right)}^{2} .
$$

因为函数 $R_{\lambda, \ell}^{\mu, j}(\xi, t)$ 关于变量 $\xi$ 的支集包含在 $\lambda^{\varepsilon} \mathcal{R}_{\lambda, \ell}^{\mu, j}$ 内, 且相函数满足非退化性质, 我们推出, 对固 定的 $\lambda^{\varepsilon} \mathcal{R}_{\lambda, \ell}^{\mu, j}$, 至多存在 $\approx \lambda^{\varepsilon}$ 同形集合与之相交. 利用该有限重叠性质, 在不计快速衰减项的情形下, 成立

$$
\left\|\sum_{\left(\ell, \ell^{\prime}\right) \in \Lambda_{j}}\left(T_{\lambda}^{j, \ell} f\right)\left(T_{\lambda}^{j, \ell^{\prime}} f\right)\right\|_{L^{\frac{5}{3}}\left(\mathbb{R}_{x, t}^{2+1}\right)} \lesssim\left(\sum_{\left(\ell, \ell^{\prime}\right) \in \Lambda_{j}}\left\|\left(T_{\lambda}^{j, \ell} f\right)\left(T_{\lambda}^{j, \ell^{\prime}} f\right)\right\|_{L^{\frac{5}{3}}\left(\mathbb{R}_{x, t}^{2+1}\right)}^{\frac{5}{3}}\right)^{\frac{3}{5}} .
$$

现在回归 (5.13) 的证明. 在相差一可忽略项的意义下, 仅需证明如下不等式:

$$
\begin{aligned}
& \left\|\left(T_{\lambda}^{j, \ell} f\right)\left(T_{\lambda}^{j, \ell^{\prime}} f\right)\right\|_{L^{5 / 3}\left(\mathbb{R}_{x, t}^{2+1}\right)} \\
& \quad \lesssim\left(\lambda 2^{-2 j}\right)^{\frac{1}{10}}\left\|\left(\sum_{\nu: \theta_{\nu} \subset \theta_{j, \ell}}\left|T_{\lambda}^{\nu}(f)\right|^{2}\right)^{\frac{1}{2}}\right\|_{L^{\frac{10}{3}\left(\mathbb{R}_{x, t}^{2+1}\right)}}\left\|\left(\sum_{\nu^{\prime}: \theta_{\nu^{\prime}} \subset \theta_{j, \ell^{\prime}}}\left|T_{\lambda}^{\nu^{\prime}}(f)\right|^{2}\right)^{\frac{1}{2}}\right\|_{L^{\frac{10}{3}\left(\mathbb{R}_{x, t}^{2+1}\right)}} .
\end{aligned}
$$

事实上, 把 (5.17) 代入到 (5.13), 利用 Cauchy-Schwarz 不等式和 $\ell^{2} \hookrightarrow \ell^{10 / 3}$, 在不计余项 $\operatorname{RapDec}(\lambda)$ 的情形下, 有

$$
\left\|\sum_{\left(\ell, \ell^{\prime}\right) \in \Lambda_{j}}\left(T_{\lambda}^{j, \ell} f\right)\left(T_{\lambda}^{j, \ell^{\prime}} f\right)\right\|_{L^{\frac{5}{3}}\left(\mathbb{R}_{x, t}^{2+1}\right)} \lesssim\left(\lambda 2^{-2 j}\right)^{\frac{1}{10}}\left\|\left(\sum_{\nu}\left|T_{\lambda}^{\nu}(f)\right|^{2}\right)^{\frac{1}{2}}\right\|_{L^{\frac{10}{3}}\left(\mathbb{R}_{x, t}^{2+1}\right)}^{2} .
$$

上式两边对 $j$ 求和, 得到了 (5.6).

\section{第 2 步 抛物尺度伸缩变换}

这一步将利用条件 (5.9) 完成 (5.17) 的证明. 为此需要抛物尺度变换技术和验证角向分离条件等. 设 $\eta^{j, \ell}$ 为 $\theta_{j, \ell}$ 的中心, 令 $\alpha_{j}^{\ell}=\eta_{1}^{j, \ell} / \eta_{2}^{j, \ell}$. 对任意 $\eta \in \theta_{j, \ell}$, 容易看出

$$
\left|\frac{\eta_{1}}{\eta_{2}}-\alpha_{j}^{\ell}\right| \leqslant 2^{-j} .
$$


通过变量替换 $\eta_{1} \rightarrow \alpha_{j}^{\ell} \eta_{2}+\eta_{1}$, 把 $\theta_{j, \ell}$ 的中心旋转到坐标轴 $\boldsymbol{e}_{2}$ 上. 相应地对空间变量 $x$ 作如下变量 替换:

$$
\left\{\begin{array}{l}
x_{1}+\alpha_{j}^{\ell} t=x_{1}^{(1)}, \\
\alpha_{j}^{\ell} x_{1}+x_{2}+\frac{1}{2}\left(\alpha_{j}^{\ell}\right)^{2} t=x_{2}^{(1)}, \\
t=t^{(1)}
\end{array}\right.
$$

其中 $x^{(1)}:=\left(x_{1}^{(1)}, x_{2}^{(1)}\right)$. 注意到变换 $(5.18)$ 是微分同胚, 用 $\Phi^{(1)}\left(x^{(1)}, t^{(1)}\right)$ 表示 $(5.18)$ 中的逆映射. 在 新坐标系下, 相函数 $\phi$ 变换成

$$
\phi^{(1)}\left(x^{(1)}, t^{(1)}, \eta\right)=\left\langle x^{(1)}, \eta\right\rangle+\frac{1}{2} t^{(1)} \frac{\eta_{1}^{2}}{\eta_{2}}+\eta_{2} \mathcal{E}_{1}\left(x^{(1)}, t^{(1)}, \frac{\eta_{1}}{\eta_{2}}+\alpha_{j}^{\ell}\right)
$$

其中

$$
\mathcal{E}_{1}\left(x^{(1)}, t^{(1)}, \frac{\eta_{1}}{\eta_{2}}+\alpha_{j}^{\ell}\right)=\mathcal{E}\left(\Phi^{(1)}\left(x^{(1)}, t^{(1)}\right), \frac{\eta_{1}}{\eta_{2}}+\alpha_{j}^{\ell}\right)
$$

然后, 对 $\mathcal{E}_{1}\left(x^{(1)}, t^{(1)}, \eta_{1} / \eta_{2}+\alpha_{j}^{\ell}\right)$ 按照如下方式进行 Taylor 展开:

$$
\begin{aligned}
\mathcal{E}_{1}\left(x^{(1)}, t^{(1)}, \frac{\eta_{1}}{\eta_{2}}+\alpha_{j}^{\ell}\right)= & \mathcal{E}_{1}\left(x^{(1)}, t^{(1)}, \alpha_{j}^{\ell}\right)+\partial_{s} \mathcal{E}_{1}\left(x^{(1)}, t^{(1)}, \alpha_{j}^{\ell}\right) \frac{\eta_{1}}{\eta_{2}} \\
& +\frac{1}{2} \partial_{s}^{2} \mathcal{E}_{1}\left(x^{(1)}, t^{(1)}, \alpha_{j}^{\ell}\right)\left(\frac{\eta_{1}}{\eta_{2}}\right)^{2} \\
& +\frac{1}{2} \int_{0}^{1} \partial_{s}^{3} \mathcal{E}_{1}\left(x^{(1)}, t^{(1)}, s \frac{\eta_{1}}{\eta_{2}}+\alpha_{j}^{\ell}\right)\left(\frac{\eta_{1}}{\eta_{2}}\right)^{3}(1-s)^{2} d s .
\end{aligned}
$$

利用坐标变换

$$
\left\{\begin{array}{l}
x_{1}^{(1)}+\partial_{s} \mathcal{E}_{1}\left(x^{(1)}, t^{(1)}, \alpha_{j}^{\ell}\right)=x_{1}^{(2)}, \\
x_{2}^{(1)}+\mathcal{E}_{1}\left(x^{(1)}, t^{(1)}, \alpha_{j}^{\ell}\right)=x_{2}^{(2)}, \\
\frac{1}{2} t^{(1)}+\frac{1}{2} \partial_{s}^{2} \mathcal{E}_{1}\left(x^{(1)}, t^{(1)}, \alpha_{j}^{\ell}\right)=\frac{1}{2} t^{(2)},
\end{array}\right.
$$

用 $\Phi^{(2)}\left(x^{(2)}, t^{(2)}\right)$ 表示 (5.20) 中的逆映射, (5.19) 中的相函数变成

$$
\phi^{(2)}\left(x^{(2)}, t^{(2)}, \eta\right)=\left\langle x^{(2)}, \eta\right\rangle+\frac{1}{2} t^{(2)} \frac{\eta_{1}^{2}}{\eta_{2}}+\mathcal{E}_{2}\left(x^{(2)}, t^{(2)}, \frac{\eta_{1}}{\eta_{2}}\right),
$$

其中

$$
\mathcal{E}_{2}\left(x^{(2)}, t^{(2)}, \frac{\eta_{1}}{\eta_{2}}\right)=\frac{1}{2} \int_{0}^{1} \partial_{s}^{3} \mathcal{E}\left(\Phi^{(1)} \circ \Phi^{(2)}\left(x^{(2)}, t^{(2)}\right), s \frac{\eta_{1}}{\eta_{2}}+\alpha_{j}^{\ell}\right)\left(\frac{\eta_{1}}{\eta_{2}}\right)^{3}(1-s)^{2} d s .
$$

通过伸缩变换 $\eta_{1} \rightarrow 2^{-j} \eta_{1}$, 相应的振幅函数变成

$$
\begin{aligned}
& a_{2}^{j, \ell}\left(x^{(2)}, t^{(2)}, \eta\right)=a\left(\Phi^{(1)} \circ \Phi^{(2)}\left(x^{(2)}, t^{(2)}\right), 2^{-j} \eta_{1}+\alpha_{j}^{\ell} \eta_{2}, \eta_{2}\right) \chi_{j, \ell}\left(\alpha_{j}^{\ell} \eta_{2}+2^{-j} \eta_{1}, \eta_{2}\right), \\
& a_{2}^{j, \ell, \ell^{\prime}}\left(x^{(2)}, t^{(2)}, \eta\right)=a\left(\Phi^{(1)} \circ \Phi^{(2)}\left(x^{(2)}, t^{(2)}\right), 2^{-j} \eta_{1}+\alpha_{j}^{\ell} \eta_{2}, \eta_{2}\right) \chi_{j, \ell^{\prime}}\left(\alpha_{j}^{\ell} \eta_{2}+2^{-j} \eta_{1}, \eta_{2}\right),
\end{aligned}
$$


其中二者关于变量 $\eta$ 的支集分别包含在

$$
\left\{\begin{array}{l}
D_{1}=\left\{\eta:\left|\frac{\eta_{1}}{\eta_{2}}\right| \leqslant \frac{\varepsilon_{0}}{4},\left|\eta_{2}-1\right| \leqslant \varepsilon_{0}\right\}, \\
D_{2}=\left\{\eta^{\prime}:\left|\frac{\eta_{1}^{\prime}}{\eta_{2}^{\prime}}-\alpha_{j}^{\ell, \ell^{\prime}}\right| \leqslant \frac{\varepsilon_{0}}{4},\left|\eta_{2}^{\prime}-1\right| \leqslant \varepsilon_{0}\right\}
\end{array}\right.
$$

中, 这里

$$
\alpha_{j}^{\ell, \ell^{\prime}}=2^{j}\left(\frac{\eta_{1}^{j, \ell^{\prime}}}{\eta_{2}^{j, \ell^{\prime}}}-\frac{\eta_{1}^{j, \ell}}{\eta_{2}^{j, \ell}}\right) .
$$

容易看出 $\left|\alpha_{j}^{\ell, \ell^{\prime}}\right| \geqslant \varepsilon_{0}$, 且 $\left|\alpha_{j}^{\ell, \ell^{\prime}}\right|$ 不依赖于 $j 、 \ell$ 和 $\ell^{\prime}$. 因此, (5.17) 的证明归结为证明

$$
\begin{aligned}
& \left\|\mathcal{T}_{\lambda}^{j, \ell} f \mathcal{T}_{\lambda}^{j, \ell, \ell^{\prime}} f\right\|_{L^{\frac{5}{3}}\left(\mathbb{R}^{3}\right)} \\
& \quad \lesssim\left(\lambda 2^{-2 j}\right)^{\frac{1}{10}}\left\|\left(\sum_{\nu: \theta_{\nu} \subset \theta_{j, \ell}}\left|\mathcal{T}_{\lambda}^{\nu} f\right|^{2}\right)^{\frac{1}{2}}\right\|\left\|_{L^{\frac{10}{3}}\left(\mathbb{R}^{3}\right)}\right\|\left(\sum_{\nu^{\prime}: \theta_{\nu^{\prime}} \subset \theta_{j, \ell^{\prime}}}\left|\mathcal{T}_{\lambda}^{\nu^{\prime}} f\right|^{2}\right)^{\frac{1}{2}} \|_{L^{\frac{10}{3}}\left(\mathbb{R}^{3}\right)},
\end{aligned}
$$

其中

$$
\begin{aligned}
& \mathcal{T}_{\lambda}^{j, \ell} f=\sum_{\nu: \theta_{\nu} \subset \theta_{j, \ell}} \mathcal{T}_{\lambda}^{\nu} f, \quad \mathcal{T}_{\lambda}^{j, \ell, \ell^{\prime}} f=\sum_{\nu: \theta_{\nu}, \subset \theta_{j, \ell^{\prime}}} \mathcal{T}_{\lambda}^{\nu^{\prime}} f, \\
& \mathcal{T}_{\lambda}^{j, \ell} f\left(x^{(2)}, t^{(2)}\right)=\int \mathrm{e}^{\mathrm{i} \lambda \phi^{(3)}\left(x^{(2)}, t^{(2)}, \eta\right)} a_{2}^{j, \ell}\left(x^{(2)}, t^{(2)}, \eta\right) f(\eta) d \eta, \\
& \mathcal{T}_{\lambda}^{j, \ell, \ell^{\prime}} f\left(x^{(2)}, t^{(2)}\right)=\int \mathrm{e}^{\mathrm{i} \lambda \phi^{(3)}\left(x^{(2)}, t^{(2)}, \eta\right)} a_{2}^{j, \ell, \ell^{\prime}}\left(x^{(2)}, t^{(2)}, \eta\right) f(\eta) d \eta, \\
& \mathcal{T}_{\lambda}^{\nu} f\left(x^{(2)}, t^{(2)}\right)=\int \mathrm{e}^{\mathrm{i} \lambda \phi^{(3)}\left(x^{(2)}, t^{(2)}, \eta\right)} a_{2}^{\nu}\left(x^{(2)}, t^{(2)}, \eta\right) f(\eta) d \eta, \\
& a_{2}^{\nu}\left(x^{(2)}, t^{(2)}, \eta\right)=a\left(\Phi^{(1)} \circ \Phi^{(2)}\left(x^{(2)}, t^{(2)}\right), 2^{-j} \eta_{1}+\alpha_{j}^{\ell} \eta_{2}, \eta_{2}\right) \chi_{\nu}\left(\alpha_{j}^{\ell} \eta_{2}+2^{-j} \eta_{1}, \eta_{2}\right),
\end{aligned}
$$

相函数为

$$
\phi^{(3)}\left(x^{(2)}, t^{(2)}, \eta\right)=2^{-j} x_{1}^{(2)} \eta_{1}+x_{2}^{(2)} \eta_{2}+2^{-2 j} t^{(2)} \eta_{1}^{2}\left(2 \eta_{2}\right)^{-1}+\eta_{2} \mathcal{E}_{2}\left(x^{(2)}, t^{(2)}, 2^{-j} \frac{\eta_{1}}{\eta_{2}}\right) .
$$

设 $\left\{R_{\mu}\right\}_{\mu}$ 是尺度 $\sim 2^{-j} \times 2^{-2 j}$ 的长方形所构成的集合, 要求这些长方形集合覆盖球 $B\left(0, \varepsilon_{0}\right) \subset \mathbb{R}^{2}$. 对每个 $\mu$, 用 $x_{\mu}$ 表示 $R_{\mu}$ 的中心, $R_{\mu}^{\varepsilon_{0}}=R_{\mu} \times\left(-\varepsilon_{0}, \varepsilon_{0}\right)$. 对 $B\left(0, \varepsilon_{0}\right) \subset \mathbb{R}^{3}$, 有

$$
B_{\varepsilon_{0}}:=B\left(0, \varepsilon_{0}\right) \subset \bigcup_{\mu} R_{\mu}^{\varepsilon_{0}}
$$

因此, 对每个 $R_{\mu}^{\varepsilon_{0}}$, 仅需证明

$$
\begin{aligned}
& \left\|\mathcal{T}_{\lambda}^{j, \ell} f \mathcal{T}_{\lambda}^{j, \ell, \ell^{\prime}} f\right\|_{L^{\frac{5}{3}}\left(R_{\mu}^{\varepsilon_{0}}\right)} \\
& \quad \lesssim\left(\lambda 2^{-2 j}\right)^{\frac{1}{10}}\left\|\left(\sum_{\nu: \theta_{\nu} \subset \theta_{j, \ell}}\left|\mathcal{T}_{\lambda}^{\nu} f\right|^{2}\right)^{\frac{1}{2}}\right\|_{L^{\frac{10}{3}}\left(w_{R_{\mu}^{\varepsilon}}^{\varepsilon_{0}}\right)}\left\|\left(\sum_{\nu^{\prime}: \theta_{\nu} \subset \theta_{j, \ell^{\prime}}}\left|\mathcal{T}_{\lambda}^{\nu^{\prime}} f\right|^{2}\right)^{\frac{1}{2}}\right\|_{L^{\frac{10}{3}}\left(w_{\left.R_{\mu}^{\varepsilon_{0}}\right)}\right.},
\end{aligned}
$$

其中隐形常数关于 $\mu$ 是一致有界的. 事实上, 注意到

$$
\sum_{\mu} w_{R_{\mu}^{\varepsilon_{0}}}=w_{B_{\varepsilon_{0}}}
$$


(5.26) 的两边对所有 $\mu$ 进行求和, 利用 Cauchy-Schwarz 不等式, 我们得到 (5.23).

通过变量替换 $x^{(2)} \rightarrow x_{\mu}+\left(2^{-j} \tilde{x}_{1}, 2^{-2 j} \tilde{x}_{2}\right), t \rightarrow \tilde{t},(5.26)$ 可归结为证明

$$
\begin{aligned}
& \left\|\tilde{\mathcal{T}}_{\lambda 2^{-2 j}}^{j, \ell} f \tilde{\mathcal{T}}_{\lambda 2^{-2 j}}^{j, \ell \ell^{\prime}} f\right\|_{L^{\frac{5}{3}}\left(B_{\varepsilon_{0}}\right)} \\
& \lesssim\left(\lambda 2^{-2 j}\right)^{\frac{1}{10}}\left\|\left(\sum_{\nu: \theta_{\nu} \subset \theta_{j, \ell}}\left|\tilde{\mathcal{T}}_{\lambda 2^{2}-2 j}^{\nu} f\right|^{2}\right)^{\frac{1}{2}}\right\|_{L^{\frac{10}{3}}\left(w_{B_{\varepsilon_{0}}}\right)}\left\|\left(\sum_{\nu^{\prime}: \theta_{\nu^{\prime}} \subset \theta_{j, \ell^{\prime}}}\left|\tilde{\mathcal{T}}_{\lambda 2^{-2 j}}^{\nu^{\prime}} f\right|^{2}\right)^{\frac{1}{2}}\right\|_{L^{\frac{10}{3}}\left(w_{B_{\varepsilon_{0}}}\right)},
\end{aligned}
$$

其中 $\tilde{\mathcal{T}}_{\lambda 2^{-2 j}}^{j, \ell} f$ 和 $\tilde{\mathcal{T}}_{\lambda 2^{-2 j}}^{j, \ell \ell^{\prime}} f$ 中的相函数和振幅函数分别为

$$
\left\{\begin{array}{l}
\tilde{\phi}^{j}(\tilde{x}, \tilde{t}, \eta)=\tilde{x} \eta+\frac{1}{2} \tilde{t} \frac{\eta_{1}^{2}}{\eta_{2}}+2^{2 j} \eta_{2} \mathcal{E}_{2}\left(x_{\mu}+\left(2^{-j} \tilde{x}_{1}, 2^{-2 j} \tilde{x}_{2}\right), t, 2^{-j} \frac{\eta_{1}}{\eta_{2}}\right), \\
\tilde{a}^{j, \ell}(\tilde{x}, \tilde{t}, \eta)=a_{2}^{j, \ell}\left(x_{\mu}+\left(2^{-j} \tilde{x}_{1}, 2^{-2 j} \tilde{x}_{2}\right), \tilde{t}, \eta\right), \\
\tilde{a}^{j, \ell, \ell^{\prime}}(\tilde{x}, \tilde{t}, \eta)=a_{2}^{j, \ell, \ell^{\prime}}\left(x_{\mu}+\left(2^{-j} \tilde{x}_{1}, 2^{-2 j} \tilde{x}_{2}\right), \tilde{t}, \eta\right) .
\end{array}\right.
$$

我们将会看到 (4.10) 中的常数不依赖于 $j$, 有限多的 $A_{\beta}$ 是一致有界的. 注意到余项 $\mathcal{E}_{2}$ 在

$$
\left\|\partial_{z}^{\beta} \tilde{\phi}^{j}-\partial_{z}^{\beta} \phi_{\infty}\right\|_{L^{\infty}} \rightarrow 0, \quad j \rightarrow \infty, \quad|\beta| \leqslant N
$$

意义下收敛, 从 (5.22) 容易得到

$$
\tilde{\phi}_{\infty}=x \eta+\frac{t}{2} \frac{\eta_{1}^{2}}{\eta_{2}} .
$$

利用 (5.9), 只需把 $\lambda$ 替换成 $\lambda 2^{-2 j}$, 就能得到 (5.27).

\section{2 双线性振荡积分的应用}

根据前面讨论, 从命题 5.1 推出 (5.6). 因此, 本小节的主要任务是证明 (5.9). 证明的方式是双线 性振荡积分估计技术. 定义伸缩函数 $\phi^{\lambda}$ 和 $a_{\lambda}$ 分别为

$$
\phi^{\lambda}(z, \eta):=\lambda \phi\left(\frac{z}{\lambda}, \eta\right), \quad a_{\lambda}(z, \eta):=a\left(\frac{z}{\lambda}, \eta\right) .
$$

相应地定义 $\mathscr{T}_{\lambda}$ 为

$$
\left(\mathscr{T}_{\lambda} f\right)(z):=\left(T_{\lambda} f\right)\left(\frac{z}{\lambda}\right)=\int \mathrm{e}^{\mathrm{i} \phi^{\lambda}(x, t, \eta)} a_{\lambda}(x, t, \eta) f(\eta) d \eta .
$$

按照上述的方法做角向分解, $\mathscr{T}_{\lambda} f$ 可以写成

$$
\begin{aligned}
& \mathscr{T}_{\lambda} f(z)=\sum_{\nu} \mathscr{T}_{\lambda}^{\nu} f(z), \\
& \mathscr{T}_{\lambda}^{\nu} f(z)=\int \mathrm{e}^{\mathrm{i} \phi^{\lambda}(x, t, \eta)} a_{\lambda}^{\nu}(x, t, \eta) f(\eta) d \eta,
\end{aligned}
$$

其中振幅函数 $a_{\lambda}^{\nu}$ 定义为 $a_{\lambda}^{\nu}(z, \eta)=a_{\lambda}(z, \eta) \chi_{\nu}(\eta)$. 在伸缩后的相函数和振幅函数的框架下, 我们可以 重新表述 (5.9) 如下:

命题 5.2 设 $\left(\Omega, \Omega^{\prime}\right)$ 满足 (5.8) 中的角向分离条件. 在不计可忽略的余项的情形下, 有

$$
\begin{aligned}
& \left\|\sum_{\nu \in \Omega} \mathscr{T}_{\lambda}^{\nu} f \sum_{\nu^{\prime} \in \Omega^{\prime}} \mathscr{T}_{\lambda}^{\nu^{\prime}} f\right\|_{L^{\frac{5}{3}}\left(\mathbb{R}^{2+1}\right)} \\
& \quad \lesssim \lambda^{\frac{1}{10}}\left\|\left(\sum_{\nu \in \Omega}\left|\mathscr{T}_{\lambda}^{\nu} f\right|^{2}\right)^{\frac{1}{2}}\right\|_{L^{\frac{10}{3}}\left(\mathbb{R}^{2+1}\right)}\left\|\left(\sum_{\nu^{\prime} \in \Omega^{\prime}}\left|\mathscr{T}_{\lambda}^{\nu^{\prime}} f\right|^{2}\right)^{\frac{1}{2}}\right\|_{L^{\frac{10}{3}}\left(\mathbb{R}^{2+1}\right)} .
\end{aligned}
$$


定义 5.1 (局部常数性质) 对于 $n \geqslant 1$, 给定函数 $F: \mathbb{R}^{n} \rightarrow[0, \infty)$. 如果 $|x-y| \leqslant C_{0} \rho$, 就有 $F(x) \approx F(y)$, 其中, $\approx$ 中的隐性常数可以依赖结构常数 $C_{0}$, 则称 $F$ 在尺度 $\rho$ 上满足局部常数性质.

定义延拓性算子

$$
E f(x, t):=\int_{\mathbb{R}^{2}} \mathrm{e}^{\mathrm{i}(x \eta+t h(\eta))} a_{2}(\eta) f(\eta) d \eta,
$$

其中 $h(\eta)$ 是齐次度为 1 并在原点之外光滑的函数, 满足

$$
\operatorname{rank} \partial_{\eta \eta}^{2} h=1 \text {, 对所有的 } \eta \in \operatorname{supp} a_{2} \text {. }
$$

对于 $r \geqslant 1$, 如果 $f$ 是支撑在 $\eta_{0} \in \operatorname{supp} a_{2}$ 的 $r^{-1}$ 邻域内, 则 $\operatorname{supp} \widehat{E f}$ 的支集同样包含在半径为 $r^{-1}$ 的球内. 由不确定性原理, 可粗略认为 $|E f|$ 在尺度为 $r$ 的球内满足局部常数性质. 然而对振荡积 分算子 $\mathscr{T}_{\lambda}$ 而言, 上述性质未必成立. 虽然如此, 在不计相旋转及可忽略余项意义下, 仍可重新获得局 部常数性质.

引理 5.1 ${ }^{[49]}$ 对 (5.32) 定义的算子 $\mathscr{T}_{\lambda}$ 而言, 一定存在一个快速衰减的光滑函数 $\varrho: \mathbb{R}^{3} \rightarrow[0, \infty)$ : supp $\hat{\varrho} \subset B(0,1)$, 使得对任意 $\varepsilon>0,1 \leqslant r \leqslant \lambda^{1-\varepsilon}, f$ 支撑在一个中心为 $\bar{\eta}$ 、尺度为 $r^{-1}$ 的正方形内， 对所有 $z \in \mathbb{R}^{3}$,

$$
\mathrm{e}^{-\mathrm{i} \phi^{\lambda}(z, \bar{\eta})} \mathscr{T}_{\lambda} f(z)=\left(\left[\mathrm{e}^{-\mathrm{i} \phi^{\lambda}(\cdot, \bar{\eta})} \mathscr{T}_{\lambda} f(\cdot)\right] * \varrho_{r}\right)(z)+\operatorname{RapDec}(\lambda)\|f\|_{L^{\frac{10}{3}}\left(\mathbb{R}^{2}\right)}
$$

成立, 其中 $\varrho_{r}(z)=r^{-3} \varrho(z / r)$.

注 5.2 选取合适的函数 $\varrho$ 使其在尺度为 1 的球上满足局部常数性质. 我们可以视 $\varrho_{r}$ 在尺度为 $r$ 的球上为常数.

证明 由 $\operatorname{supp} f \subset B\left(\bar{\eta}, r^{-1}\right)$, 有

$$
\left[\mathrm{e}^{-\mathrm{i} \phi^{\lambda}(\cdot, \bar{\eta})} \mathscr{T}_{\lambda} f\right]^{\wedge}(\xi)=\int_{\mathbb{R}^{2}} K^{\lambda}(\xi, \eta) f(\eta) d \eta,
$$

其中核函数 $K^{\lambda}$ 可表示为

$$
K^{\lambda}(\xi, \eta)=\lambda^{3} \int_{\mathbb{R}^{3}} \mathrm{e}^{-\mathrm{i} \lambda(\langle z, \xi\rangle)-\phi(z, \eta)+\phi(z, \bar{\eta})} a(z, \eta) d z .
$$

注意到

$$
\left|\partial_{z} \phi(z, \eta)-\partial_{z} \phi(z, \bar{\eta})\right| \lesssim r^{-1}, \quad(z, \eta) \in \operatorname{supp}_{z, \eta} a(z, \eta),
$$

根据驻相分析方法, 若 $|\xi| \geqslant r^{-1}$, 则利用分部积分, 可得

$$
\left|K^{\lambda}(\xi, \eta)\right| \leqslant \lambda^{-N}(1+|\xi|)^{-4}
$$

设 $\varrho$ 为 $\mathbb{R}^{3}$ 上的 Schwartz 函数, 满足对于 $|\xi| \leqslant C$ 有 $\varrho(\xi)=1$. 由 (5.38), 得到

$$
\left[\mathrm{e}^{-\mathrm{i} \phi^{\lambda}(\cdot, \bar{\eta})} \mathscr{T}_{\lambda} f\right]^{\wedge}(\xi) \hat{\varrho}_{r}(\xi)+\lambda^{-N}(1+|\xi|)^{-(n+1)}\|f\|_{L^{\frac{10}{3}}\left(\mathbb{R}^{2}\right)},
$$

由此推出 (5.37).

为了证明命题 5.2 , 我们需要充分开发局部常数性质. 为此, 对映射 $\eta \rightarrow a_{\lambda}^{\nu}(z, \eta)$ 的支集进行径向 分解. 设 $\rho \in C_{c}^{\infty}\left(\mathbb{R}^{2}\right)$ 满足

$$
\sum_{j \in \mathbb{Z}^{2}} \rho(\eta-j) \equiv 1, \quad \eta \in \mathbb{R}^{2}
$$


令 $\varepsilon>0$ 足够小, $\mathbf{Q}=\left\{Q_{k}\right\}_{k}$ 是一组边长为 $\lambda^{1 / 2-\varepsilon}$ 的方体, 方体中心落在格点 $\lambda^{1 / 2-\varepsilon} \mathbb{Z}^{2+1}$ 上. 记每个 $Q_{k} \in \mathbf{Q}$ 的中心为 $z_{k}$, 令

$$
\begin{aligned}
& \mathscr{T}_{\lambda, k}^{\nu, j} f(z)=\int \mathrm{e}^{\mathrm{i} \phi^{\lambda}(z, \eta)} a_{\lambda, k}^{\nu, j}(z, \eta) f(\eta) d \eta, \\
& a_{\lambda, k}^{\nu, j}(z, \eta)=a_{\lambda}^{\nu}(z, \eta) \rho\left(\lambda^{\frac{1}{2}-\frac{\varepsilon}{2}} \partial_{x} \phi^{\lambda}\left(z_{k}, \eta\right)-j\right),
\end{aligned}
$$

则 $\eta \rightarrow a_{\lambda, k}^{\nu, j}(z, \cdot)$ 的支集是包含在一个边长 $\approx \lambda^{-1 / 2+\varepsilon / 2}$ 的方体内, 记该方体为 $\mathcal{D}_{k}^{\nu, j}$. 我们得到

$$
\left.\mathscr{T}_{\lambda} f(z)\right|_{z \in Q_{k}}=\sum_{\nu, j} \mathscr{T}_{\lambda, k}^{\nu, j} f(z),\left.\quad \mathscr{T}_{\lambda}^{\nu} f(z)\right|_{z \in Q_{k}}=\sum_{j} \mathscr{T}_{\lambda, k}^{\nu, j} f(z) .
$$

设 $\eta_{k}^{\nu, j}$ 是 $\mathcal{D}_{k}^{\nu, j}$ 的中心, 余下部分固定 $R=\lambda^{1 / 2-\varepsilon / 2}$, 则证明命题 5.2 的关键是建立如下的离散双线性 平方函数估计.

命题 5.3 设 $Q_{k} \in \mathbf{Q},\left(\nu, \nu^{\prime}\right) \in \Omega \times \Omega^{\prime}$ 满足 (5.8) 中的角向分离条件, 则

$$
\left\|\sum_{\nu, j} \mathrm{e}^{\mathrm{i} \phi^{\lambda}\left(z, \eta_{k}^{\nu, j}\right)} c^{\nu, j} \sum_{\nu^{\prime}, j^{\prime}} \mathrm{e}^{\mathrm{i} \phi^{\lambda}\left(z, \eta_{k}^{\nu^{\prime}, j^{\prime}}\right)} c^{\nu^{\prime}, j^{\prime}}\right\|_{L^{\frac{5}{3}}\left(Q_{k}\right)} \lesssim \lambda\left(\sum_{\nu, j}\left|c^{\nu, j}\right|^{2}\right)^{\frac{1}{2}}\left(\sum_{\nu^{\prime}, j^{\prime}}\left|c^{\nu^{\prime}, j^{\prime}}\right|^{2}\right)^{\frac{1}{2}} .
$$

证明 不失一般性, 假设 $z_{k}=\mathbf{0}$, 通过变换 $\psi^{\lambda}(z, \eta)=\phi^{\lambda}(z, \eta)-\phi^{\lambda}(\mathbf{0}, \eta)$ 规范化相函数. 设

$$
b_{k}^{\nu, j}(z, \eta)=\left(\int_{\mathcal{D}_{k}^{\nu, j}} \mathrm{e}^{\mathrm{i}\left[\psi^{\lambda}(z, \eta)-\psi^{\lambda}\left(z, \eta_{k}^{\nu, j}\right)\right]} d \eta\right)^{-1} \chi_{\mathcal{D}_{k}^{\nu, j}}(\eta),
$$

其中 $\chi_{\mathcal{D}_{k}^{\nu, j}}$ 表示 $\mathcal{D}_{k}^{\nu, j}$ 的特征函数. 容易看出, 对于充分大的 $\lambda$, 有

$$
\left|b_{k}^{\nu, j}(z, \eta)\right| \leqslant R^{2}, \quad\left|\partial_{z}^{\alpha} b_{k}^{\nu, j}(z, \eta)\right| \leqslant R^{2-|\alpha|}, \quad \forall z \in Q_{k} .
$$

我们的目标转化为估计

$$
\left\|\left(\int \mathrm{e}^{\mathrm{i} \psi^{\lambda}(z, \eta)} \sum_{\nu, j} b_{k}^{\nu, j}(z, \eta) c^{\nu, j} d \eta\right)\left(\int \mathrm{e}^{\mathrm{i} \psi^{\lambda}\left(z, \eta^{\prime}\right)} \sum_{\nu^{\prime}, j^{\prime}} b_{k}^{\nu^{\prime}, j^{\prime}}\left(z, \eta^{\prime}\right) c^{\nu^{\prime}, j^{\prime}} d \eta^{\prime}\right)\right\|_{L^{\frac{5}{3}}\left(Q_{k}\right)} .
$$

设

$$
B_{k}\left(z, \eta, \eta^{\prime}\right)=\sum_{\nu, j} \sum_{\nu^{\prime}, j^{\prime}} c^{\nu, j} b_{k}^{\nu, j}(z, \eta) c^{\nu^{\prime}, j^{\prime}} b_{k}^{\nu^{\prime}, j^{\prime}}\left(z, \eta^{\prime}\right),
$$

由微积分基本定理, 可以得到

$$
\begin{aligned}
B_{k}\left(z, \eta, \eta^{\prime}\right)= & B_{k}\left(\mathbf{0}, \eta, \eta^{\prime}\right)+\int_{0}^{x_{1}} \frac{\partial B_{k}}{\partial u_{1}}\left(\left(u_{1}, 0,0\right), \eta, \eta^{\prime}\right) d u_{1} \\
& +\cdots+\int_{0}^{x_{1}} \int_{0}^{x_{2}} \int_{0}^{t} \frac{\partial^{3} B_{k}}{\partial u_{1} \partial u_{2} \partial u_{3}}\left(u, \eta, \eta^{\prime}\right) d u .
\end{aligned}
$$

选取 $B_{k}\left(\mathbf{0}, \eta, \eta^{\prime}\right)$ 作为例子, 其他情形的证明是类似的. 注意到

$$
B_{k}\left(\mathbf{0}, \eta, \eta^{\prime}\right)=\sum_{\nu, j} c^{\nu, j} b_{k}^{\nu, j}(\mathbf{0}, \eta) \sum_{\nu^{\prime}, j^{\prime}} c^{\nu^{\prime}, j^{\prime}} b_{k}^{\nu^{\prime}, j^{\prime}}\left(\mathbf{0}, \eta^{\prime}\right)
$$

于是仅需估计

$$
\left\|\left(\int \mathrm{e}^{\mathrm{i} \psi^{\lambda}(z, \eta)} \sum_{\nu, j} c^{\nu, j} b_{k}^{\nu, j}(\mathbf{0}, \eta) d \eta\right)\left(\int \mathrm{e}^{\mathrm{i} \psi^{\lambda}\left(z, \eta^{\prime}\right)} \sum_{\nu^{\prime}, j^{\prime}} c^{\nu^{\prime}, j^{\prime}} b_{k}^{\nu^{\prime}, j^{\prime}}\left(\mathbf{0}, \eta^{\prime}\right) d \eta^{\prime}\right)\right\|_{L^{\frac{5}{3}}} .
$$


根据定理 4.1 , 假设相函数 $\psi^{\lambda}(z, \eta)$ 和 $\psi^{\lambda}\left(z, \eta^{\prime}\right)$ 满足 (4.10), 则 (5.48) 可以被下式控制:

$$
\left\|\sum_{\nu, j} c^{\nu, j} b_{k}^{\nu, j}(\mathbf{0}, \cdot)\right\|_{L^{2}}\left\|\sum_{\nu^{\prime}, j^{\prime}} c^{\nu^{\prime}, j^{\prime}} b_{k}^{\nu^{\prime}, j^{\prime}}(\mathbf{0}, \cdot)\right\|_{L^{2}} \lesssim C \lambda\left(\sum_{\nu, j}\left|c^{\nu, j}\right|^{2}\right)^{\frac{1}{2}}\left(\sum_{\nu^{\prime}, j^{\prime}}\left|c^{\nu^{\prime}, j^{\prime}}\right|^{2}\right)^{\frac{1}{2}} .
$$

下面利用角向分离条件 (5.8) 验证假设条件 (4.10). 事实上, 通过变量替换 $z \rightarrow \lambda z$, 仅需要验证 $\eta$ 和 $\eta^{\prime}$ 满足 $(5.8)$, 则 $\psi(z, \eta)$ 和 $\psi\left(z, \eta^{\prime}\right)$ 就满足 (4.10), 其中

$$
\psi(x, t, \eta)=\phi(x, t, \eta)-\phi(0, \eta)
$$

注意到 $\phi(x, t, \eta)$ 具有 $(1.14)$ 中的形式, 即

$$
\left\{\begin{array}{l}
\phi(x, t, \eta)=\langle x, \eta\rangle+\frac{t}{2}\left(\frac{\eta_{1}^{2}}{\eta_{2}}\right)+\eta_{2} \mathcal{E}\left(x, t, \frac{\eta_{1}}{\eta_{2}}\right), \\
\mathcal{E}(z, s)=O\left(|(x, t)|^{2} s^{2}+(|x|+|t|)|s|^{3}\right) .
\end{array}\right.
$$

由于 $\phi(0, \eta)$ 不依赖于 $(x, t)$, 因此可以忽略此项的影响.

为了确保 (4.10), 对变量 $\eta$ 实施角向变换来消除角向分离的尺度对 $\varepsilon_{0}$ 的依赖. 不失一般性, 可以 假设 $\operatorname{supp}_{\eta} a(z, \cdot)$ 包含 $\boldsymbol{e}_{2}$, 一般情形可以通过命题 5.1 的证明方法处理.

在变量替换 $\eta_{1} \rightarrow \varepsilon_{0} \eta_{1}$ 下, 只需考虑 $\eta$ 和 $\eta^{\prime}$ 分别包含在

$$
\begin{aligned}
& \mathscr{D}_{1}=\left\{\eta=\left(\eta_{1}, \eta_{2}\right) \in \mathbb{R}^{2}:\left|\frac{\eta_{1}}{\eta_{2}}\right| \leqslant \frac{1}{10},\left|\eta_{2}-1\right| \leqslant \varepsilon_{0}\right\}, \\
& \mathscr{D}_{2}=\left\{\eta^{\prime}=\left(\eta_{1}^{\prime}, \eta_{2}^{\prime}\right) \in \mathbb{R}^{2}:\left|\frac{\eta_{1}^{\prime}}{\eta_{2}^{\prime}}-\frac{1}{2}\right| \leqslant \frac{1}{10},\left|\eta_{2}^{\prime}-1\right| \leqslant \varepsilon_{0}\right\}
\end{aligned}
$$

中的情形, 此时相函数变成

$$
\phi(x, t, \eta)=\varepsilon_{0} x_{1} \eta_{1}+x_{2} \eta_{2}+\frac{t}{2} \varepsilon_{0}^{2} \frac{\eta_{1}^{2}}{\eta_{2}}+\eta_{2} \mathcal{E}\left(x, t, \varepsilon_{0} \frac{\eta_{1}}{\eta_{2}}\right) .
$$

设 $\left\{R_{\mu}\right\}_{\mu}$ 是一组两两互不相交的边长为 $\varepsilon_{0} \times \varepsilon_{0}^{2}$ 的长方形, 对每个 $\mu$, 用 $x_{\mu}$ 表示 $R_{\mu}$ 的中心. 为了简 单起见, 用 $R_{\mu}^{\varepsilon_{0}}$ 表示长方体 $R_{\mu} \times\left(-\varepsilon_{0}, \varepsilon_{0}\right)$.

通过变量替换 $x \rightarrow x_{\mu}+\left(\varepsilon_{0} x_{1}, \varepsilon_{0}^{2} x_{2}\right)$, 用 $\varepsilon_{0}^{2} \lambda$ 替换 $\lambda$, 不计余项的影响, 在新坐标系下, (5.50) 将转 化成

$$
\phi(x, t, \eta)=x \cdot \eta+\frac{t}{2} \frac{\eta_{1}^{2}}{\eta_{2}}+\varepsilon_{0}^{-2} \eta_{2} \mathcal{E}\left(x_{\mu}+\left(\varepsilon_{0} x_{1}, \varepsilon_{0}^{2} x_{2}\right), t, \varepsilon_{0} \frac{\eta_{1}}{\eta_{2}}\right) .
$$

注意到 $\left(x_{\mu}+\left(\varepsilon_{0} x_{1}, \varepsilon_{0}^{2} x_{2}\right), t\right) \in B\left(0,2 \varepsilon_{0}\right) \subset \mathbb{R}^{3}$ 以及 $\varepsilon_{0}$ 充分小, 直接计算得

$$
\left\{\begin{array}{l}
\nabla_{x} \phi(x, t, \eta)=\eta+O\left(\varepsilon_{0}|\eta|^{2}\right)+O\left(\varepsilon_{0}|\eta|^{3}\right), \\
\partial_{x, \eta}^{2} \phi(x, t, \eta)=\operatorname{Id}+O\left(\varepsilon_{0}|\eta|\right), \\
\partial_{t} \phi(x, t, \eta)=\frac{1}{2} \frac{\eta_{1}^{2}}{\eta_{2}}+O\left(\varepsilon_{0}|\eta|^{2}\right)+O\left(\varepsilon_{0}|\eta|^{3}\right) .
\end{array}\right.
$$

由 (4.6) 推知,

$$
\nabla_{\eta} \partial_{t} \phi(x, t, \eta)=\left(\nabla_{\eta} q\right)\left(x, t, \partial_{x} \phi(x, t, \eta)\right) \partial_{x \eta}^{2} \phi(x, t, \eta)
$$


进而,

$$
\left(\nabla_{\eta} q\right)\left(x, t, \partial_{x} \phi(x, t, \eta)\right)=\left(\frac{\eta_{1}}{\eta_{2}},-\frac{\eta_{1}^{2}}{2 \eta_{2}^{2}}\right)+O\left(\varepsilon_{0}|\eta|\right) .
$$

所以, 选取 $\varepsilon_{0}$ 充分小, 对 $\eta \in \mathscr{D}_{1}$ 和 $\eta^{\prime} \in \mathscr{D}_{2}$, 有

$$
(4.10) \text { 的左边 } \approx\left|\frac{\eta_{1}}{\eta_{2}}-\frac{\eta_{1}^{\prime}}{\eta_{2}^{\prime}}\right|^{2}+O\left(\varepsilon_{0}\right) \approx 1 \text {, }
$$

这验证了 (4.10).

现在转向命题 5.2 的证明. 对给定的 $R=\lambda^{1 / 2-\varepsilon / 2}, \varrho_{R}$ 在尺度为 $R$ 的球上可视为常数. 对于 $z \in Q_{k}$, 记

$$
\mathscr{H}_{\lambda, k}^{\nu, j} f(z)=\mathrm{e}^{-\mathrm{i} \phi^{\lambda}\left(z, \eta_{k}^{\nu, j}\right)} \mathscr{T}_{\lambda, k}^{\nu, j} f(z) .
$$

根据命题 5.1 及 $\operatorname{supp}_{\eta} a_{\lambda, k}^{\nu, j}(z, \cdot)$ 的紧支集性质, 容易看出

$$
\begin{aligned}
& \sum_{Q_{k} \in \mathbf{Q}}\left\|\sum_{\nu, j} \mathrm{e}^{\mathrm{i} \phi^{\lambda}\left(\cdot, \eta_{k}^{\nu, j}\right)}\left(\mathscr{H}_{\lambda, k}^{\nu, j} f\right) * \varrho_{R} \sum_{\nu^{\prime}, j^{\prime}} \mathrm{e}^{\mathrm{i} \phi^{\lambda}\left(\cdot, \eta_{k}^{\nu^{\prime}, j^{\prime}}\right)}\left(\mathscr{H}_{\lambda, k}^{\nu^{\prime}, j^{\prime}} f\right) * \varrho_{R}\right\|_{L^{\frac{5}{3}}\left(Q_{k}\right)}^{\frac{5}{3}} \\
& \quad \lesssim \lambda^{\frac{1}{10}}\left(\sum_{Q_{k} \in \mathbf{Q}}\left\|\left(\sum_{\nu, j}\left|\mathscr{T}_{\lambda, k}^{\nu, j} f\right|^{2}\right)^{\frac{1}{2}}\right\|_{L^{\frac{10}{3}}\left(w_{Q_{k}}\right)}^{\frac{10}{3}}\right)^{\frac{1}{2}}\left(\sum_{Q_{k} \in \mathbf{Q}}\left\|\left(\sum_{\nu^{\prime}, j^{\prime}}\left|\mathscr{T}_{\lambda, k}^{\nu^{\prime}, j^{\prime}} f\right|^{2}\right)^{\frac{1}{2}}\right\|_{\left.L^{\frac{10}{3}\left(w_{Q_{k}}\right)}\right)^{\frac{10}{3}} .}^{\frac{1}{2}} .\right.
\end{aligned}
$$

事实上, 由 Minkowski 不等式和 $\varrho_{R}$ 的局部常数性质, 可得

$$
\left\|\iint \varrho_{R}(z-y) \varrho_{R}\left(z-y^{\prime}\right)\left|\sum_{\nu, \nu^{\prime}, j, j^{\prime}} \mathrm{e}^{\mathrm{i} \phi^{\lambda}\left(z, \eta_{k}^{\nu, j}\right)} \mathscr{H}_{\lambda, k}^{\nu, j} f(y) \mathrm{e}^{\mathrm{i} \phi^{\lambda}\left(z, \eta_{k}^{\nu^{\prime}, j^{\prime}}\right)} \mathscr{H}_{\lambda, k}^{\nu^{\prime}, j^{\prime}} f\left(y^{\prime}\right)\right| d y d y^{\prime}\right\|_{L^{\frac{5}{3}}\left(Q_{k}\right)} .
$$

选取任意的 $\bar{z} \in Q_{k}$, 上式可被

$$
\iint\left\|\sum_{\nu, j} \mathrm{e}^{\mathrm{i} \phi^{\lambda}\left(z, \eta_{k}^{\nu, j}\right)} \mathscr{H}_{\lambda, k}^{\nu, j} f(y) \sum_{\nu^{\prime}, j^{\prime}} \mathrm{e}^{\mathrm{i} \phi^{\lambda}\left(z, \eta_{k}^{\nu^{\prime}, j^{\prime}}\right)} \mathscr{H}_{\lambda, k}^{\nu^{\prime}, j^{\prime}} f\left(y^{\prime}\right)\right\|_{L^{\frac{5}{3}}\left(Q_{k}\right)} \varrho_{R}(\bar{z}-y) \varrho_{R}\left(\bar{z}-y^{\prime}\right) d y d y^{\prime}
$$

控制.

利用引理 5.3 , 上式可被

$$
\lambda \iint\left(\sum_{\nu, j}\left|\mathscr{T}_{\lambda, k}^{\nu, j} f(\bar{z}-y)\right|^{2}\right)^{\frac{1}{2}}\left(\sum_{\nu^{\prime}, j^{\prime}}\left|\mathscr{T}_{\lambda, k}^{\nu^{\prime}, j^{\prime}} f\left(\bar{z}-y^{\prime}\right)\right|^{2}\right)^{\frac{1}{2}} \varrho_{R}(y) \varrho_{R}\left(y^{\prime}\right) d y d y^{\prime}
$$

控制. 通过对变量 $\bar{z}$ 在 $Q_{1}$ 上取平均并忽略余项 $\operatorname{RapDec}(\lambda)$ 的影响, (5.54) 可以被

$$
\lambda^{\frac{1}{10}} \int\left\|\left(\sum_{\nu, j}\left|\mathscr{T}_{\lambda, k}^{\nu, j} f(\bar{z}-y)\right|^{2}\right)^{\frac{1}{2}}\right\|_{L_{\bar{z}}^{\frac{10}{3}}\left(Q_{k}\right)} \varrho_{R}(y) d y \int\left\|\left(\sum_{\nu^{\prime}, j^{\prime}}\left|\mathscr{T}_{\lambda, k}^{\nu^{\prime}, j^{\prime}} f\left(\bar{z}-y^{\prime}\right)\right|^{2}\right)^{\frac{1}{2}}\right\|_{L_{\bar{z}}^{\frac{10}{3}}\left(Q_{k}\right)} \varrho_{R}\left(y^{\prime}\right) d y^{\prime}
$$

控制. 由 Hölder 不等式, 有

$$
\int\left\|\left(\sum_{\nu, j}\left|\mathscr{T}_{\lambda, k}^{\nu, j} f(\bar{z}-y)\right|^{2}\right)^{\frac{1}{2}}\right\|_{L_{\bar{z}}^{\frac{10}{3}}\left(Q_{k}\right)} \varrho_{R}(y) d y \lesssim\left(\int\left(\sum_{\nu, j}\left|\mathscr{T}_{\lambda, k}^{\nu, j} f(z)\right|^{2}\right)^{\frac{5}{3}} w_{Q_{k}}(z) d z\right)^{\frac{3}{10}},
$$

这里用到了

$$
\int_{\mathbb{R}^{3}} w_{Q_{k}}(z+y) \varrho_{R}(y) d y \lesssim w_{Q_{k}}(z) .
$$


对 $Q_{k} \in \mathbf{Q}$ 求和并应用 Cauchy-Schwarz 不等式, 可得 (5.53).

若能证明

$$
\left\|\left(\sum_{\nu, j}\left|\mathscr{T}_{\lambda, k}^{\nu, j} f\right|^{2}\right)^{\frac{1}{2}}\right\|_{L^{\frac{10}{3}\left(w_{Q_{k}}\right)}} \lesssim\left\|\left(\sum_{\nu}\left|\mathscr{T}_{\lambda}^{\nu} f\right|^{2}\right)^{\frac{1}{2}}\right\|_{L^{\frac{10}{3}\left(w_{Q_{k}}\right)}}+\operatorname{RapDec}(\lambda)\|f\|_{L^{\frac{10}{3}\left(\mathbb{R}^{2}\right)}},
$$

则意味着 (5.34) 成立, 从而完成了命题 5.2 的证明. 因此, 下面的任务就是证明 (5.55).

\section{3 径向黏合}

本小节致力于证明 (5.55). 主要想法是, 在充分小的空间尺度内, 用延拓算子 $E$ 逼近振荡积分算 子 $\mathscr{T}_{\lambda}$. 假设 $\delta>0$ 和 $1 \leqslant K \leqslant \lambda^{1 / 2-\delta}$, 对 $\phi^{\lambda}$ 在点 $\bar{z}$ 附近实施 Taylor 展开, 然后进行变量替换: $\eta \rightarrow \Psi^{\lambda}(\bar{z}, \eta):=\Psi(\bar{z} / \lambda, \eta)$, 容易推出

$$
\mathscr{T}_{\lambda} f(z)=\int_{\mathbb{R}^{2}} \mathrm{e}^{\mathrm{i}\left(\left\langle z-\bar{z}, \partial_{z} \phi^{\lambda}\left(\bar{z}, \Psi^{\lambda}(\bar{z}, \eta)\right)\right\rangle+\varepsilon_{\lambda}^{\bar{z}}(z-\bar{z}, \eta)\right)} a_{\lambda, \bar{z}}(z, \eta) f_{\bar{z}}(\eta) d \eta, \quad \text { 对于 }|z-\bar{z}| \leqslant K,
$$

其中

$$
\begin{aligned}
& f_{\bar{z}}:=\mathrm{e}^{\mathrm{i} \phi^{\lambda}\left(\bar{z}, \Psi^{\lambda}(\bar{z}, \cdot)\right)} f \circ \Psi^{\lambda}(\bar{z}, \cdot), \\
& a_{\lambda, \bar{z}}(z, \eta)=a_{\lambda}\left(z, \Psi^{\lambda}(\bar{z}, \eta)\right)\left|\operatorname{det} \partial_{\eta} \Psi^{\lambda}(\bar{z}, \eta)\right| .
\end{aligned}
$$

对于 $|v| \leqslant K$,

$$
\varepsilon_{\lambda}^{\bar{z}}(v, \eta)=\frac{1}{\lambda} \int_{0}^{1}(1-s)\left\langle\left(\partial_{z z}^{2} \phi\right)\left(\frac{\bar{z}+s v}{\lambda}, \Psi^{\lambda}(\bar{z}, \eta)\right) v, v\right\rangle d s .
$$

根据 (4.1), 对任意 $\lambda \gg 1$, 有

$$
\sup _{(v, \eta) \in B(0, K) \times \operatorname{supp}_{\eta} a_{\lambda, \bar{z}}}\left|\partial_{\eta}^{\beta} \varepsilon_{\lambda}^{\bar{z}}(v, \eta)\right| \leqslant 1, \quad \forall|v| \leqslant K,
$$

其中 $\beta \in \mathbb{N}^{2},|\beta| \leqslant N$. 鉴于 (4.2), 得到

$$
\left\langle z, \partial_{z} \phi^{\lambda}\left(\bar{z}, \Psi^{\lambda}(\bar{z}, \eta)\right)\right\rangle=x \eta+t h_{\bar{z}}(\eta), \quad h_{\bar{z}}(\eta):=\left(\partial_{t} \phi^{\lambda}\right)\left(\bar{z}, \Psi^{\lambda}(\bar{z}, \eta)\right) .
$$

注意到相函数 $a(z, \eta)=a_{1}(z) a_{2}(\eta)$ 的假设, 不计空间变量的影响, 在 $\bar{z}$ 的一个充分小的邻域上, 我们 可用延拓算子 $E_{\bar{z}}$ :

$$
E_{\bar{z}} g(z):=\int_{\mathbb{R}^{2}} \mathrm{e}^{\mathrm{i}\left(x \eta+t h_{\bar{z}}(\eta)\right)} a_{2, \bar{z}}(\eta) g(\eta) d \eta
$$

逼近 $\mathscr{T}_{\lambda}$, 其中

$$
a_{2, \bar{z}}(\eta)=a_{2}\left(\Psi^{\lambda}(\bar{z}, \eta)\right)\left|\operatorname{det} \partial_{\eta} \Psi^{\lambda}(\bar{z}, \eta)\right| .
$$

注意到 $h_{\bar{z}}(\eta)$ 是 1 次齐次函数且满足

$$
\operatorname{rank} \partial_{\eta \eta}^{2} h_{\bar{z}}=1, \quad \forall \eta \in \operatorname{supp} a_{2, \bar{z}} .
$$

考虑到 $a$ 支集的有界性和 (4.3), 我们可以假设 $\partial_{\eta \eta}^{2} h_{\bar{z}}(\eta)$ 的非零特征值不依赖于 $\bar{z}$.

为了说明 (5.55), 需要建立下面两个引理. 
引理 5.2 设 $z_{k}$ 为 $Q_{k}$ 的中心, 定义延拓算子 $E_{z_{k}}^{\nu, j}$ 如下:

$$
E_{z_{k}}^{\nu, j} g(z):=\int_{\mathbb{R}^{2}} \mathrm{e}^{\mathrm{i}\left(\langle x, \eta\rangle+t h_{z_{k}}(\eta)\right)} \rho\left(\lambda^{\frac{1}{2}-\frac{\varepsilon}{2}} \eta-j\right) a_{2, z_{k}}^{\nu}(\eta) g(\eta) d \eta,
$$

其中 $\rho$ 满足 $(5.40)$,

$$
a_{2, z_{k}}^{\nu}(\eta)=a_{2}^{\nu}\left(\Psi^{\lambda}\left(z_{k}, \eta\right)\right)\left|\operatorname{det} \partial_{\eta} \Psi^{\lambda}\left(z_{k}, \eta\right)\right|
$$

则

$$
\left\|\left(\sum_{\nu, j}\left|E_{z_{k}}^{\nu, j} g\right|^{2}\right)^{\frac{1}{2}}\right\|_{L^{\frac{10}{3}\left(w_{Q_{0}}\right)}} \lesssim\left\|\left(\sum_{\nu}\left|E_{z_{k}}^{\nu} g\right|^{2}\right)^{\frac{1}{2}}\right\|_{L^{\frac{10}{3}\left(w_{Q_{0}}\right)}}+\operatorname{RapDec}(\lambda)\|g\|_{L^{\frac{10}{3}}} .
$$

下面引理说明, 在充分小的空间区域内, 从某种意义上 $\mathscr{T}_{\lambda}$ 可视为 $E_{\bar{z}}$. 下面引理中的一个弱形式 出现在文献 [41] 中. 它对分离性不等式是适用的, 然而对于平方函数不等式是失效的. 为此需要如下 改进的点态估计版本.

引理 5.3 设 $0<\delta \leqslant 1 / 2$ 和 $1 \leqslant K \leqslant \lambda^{1 / 2-\delta}$. 假设 (4.1) 成立, 则对任意 $N$, 只要 $|v| \leqslant K$, 就有

$$
\begin{aligned}
& \left|\mathscr{T}_{\lambda} f(\bar{z}+v)\right| \leqslant\left|E_{\bar{z}} f_{\bar{z}}(v)\right|+\left(\frac{3}{\pi}\right)^{N} \sum_{\ell \in \mathbb{Z}^{2} \backslash\{0\}}|\ell|^{-N}\left|E_{\bar{z}}\left(f_{\bar{z}} \mathrm{e}^{\mathrm{i}\langle 4 \pi \ell, \cdot\rangle}\right)(v)\right|, \\
& \left|E_{\bar{z}} f_{\bar{z}}(v)\right| \leqslant\left|\mathscr{T}_{\lambda} f(\bar{z}+v)\right|+\left(\frac{3}{\pi}\right)^{N} \sum_{\ell \in \mathbb{Z}^{2} \backslash\{0\}}|\ell|^{-N}\left|\mathscr{T}_{\lambda}\left[\mathrm{e}^{\mathrm{i}\left\langle 4 \pi \ell,\left(\partial_{x} \phi^{\lambda}\right)(\bar{z}, \cdot)\right\rangle} f\right](\bar{z}+v)\right| .
\end{aligned}
$$

先承认引理 5.2 和 5.3 , 我们来完成 (5.55) 的证明. 设 $z_{k}$ 是 $Q_{k}$ 的中心, 由 (5.64) 可见

$$
\begin{aligned}
\left\|\left(\sum_{\nu, j}\left|\mathscr{T}_{\lambda, k}^{\nu, j} f\right|^{2}\right)^{\frac{1}{2}}\right\|_{L^{\frac{10}{3}}\left(w_{Q_{k}}\right)} \leqslant & \left\|\left(\sum_{\nu, j}\left|\mathscr{T}_{\lambda, k}^{\nu, j} f\right|^{2}\right)^{\frac{1}{2}} \chi_{\left\{\left|z-z_{k}\right| \leqslant \lambda^{\frac{1}{2}-\frac{\varepsilon}{2}}\right\}}\right\|_{L^{\frac{10}{3}}\left(w_{Q_{k}}\right)} \\
& +\left\|\left(\sum_{\nu, j}\left|\mathscr{T}_{\lambda, k}^{\nu, j} f\right|^{2}\right)^{\frac{1}{2}} \chi_{\left\{\left|z-z_{k}\right| \geqslant \lambda^{\frac{1}{2}-\frac{\varepsilon}{2}}\right\}}\right\|_{L^{\frac{10}{3}}\left(w_{Q_{k}}\right)} \\
\lesssim_{N} & \sum_{\ell \in \mathbb{Z}^{2}}(1+4 \pi|\ell|)^{-N}\left\|\left(\sum_{\nu, j}\left|E_{z_{k}}^{\nu, j}\left(f_{z_{k}} \mathrm{e}^{\mathrm{i}\langle 4 \pi \ell, \cdot\rangle}\right)\right|^{2}\right)^{\frac{1}{2}}\right\|_{L^{\frac{10}{3}}\left(w_{Q_{0}}\right)} \\
& +\operatorname{RapDec}(\lambda)\|f\|_{L^{\frac{10}{3}}}
\end{aligned}
$$

需要指出的是, (5.66) 中最后不等式中的正方体 $Q_{0}$ 比原先的要稍微大一些. 利用 (5.63), 在忽略余项 情形下, 有

$$
\begin{aligned}
& \sum_{\ell}(1+4 \pi|\ell|)^{-N}\left\|\left(\sum_{\nu, j}\left|E_{z_{k}}^{\nu, j}\left(f_{z_{k}} \mathrm{e}^{\mathrm{i}\langle 4 \pi \ell, \cdot\rangle}\right)\right|^{2}\right)^{\frac{1}{2}}\right\|_{L^{\frac{10}{3}}\left(w_{Q_{0}}\right)} \\
& \lesssim \sum_{\ell}(1+4 \pi|\ell|)^{-N}\left\|\left(\sum_{\nu}\left|E_{z_{k}}^{\nu}\left(f_{z_{k}} \mathrm{i} \mathrm{i}^{\mathrm{i}\langle 4 \pi \ell, \cdot\rangle}\right)\right|^{2}\right)^{\frac{1}{2}}\right\|_{L^{\frac{10}{3}}\left(w_{Q_{0}}\right)} \\
& \lesssim \sum_{\ell}(1+4 \pi|\ell|)^{-N}\left\|\left(\sum_{\nu}\left|E_{z_{k}}^{\nu} f_{z_{k}}\right|^{2}\right)^{\frac{1}{2}}\right\|_{L^{\frac{10}{3}}\left(w_{\left.Q_{0}, \ell\right)}\right.},
\end{aligned}
$$

其中

$$
w_{Q_{0}, \ell}(z)=w_{Q_{0}}((4 \pi \ell, 0)+z) .
$$


最后一个不等式用到了延拓算子 $E_{\bar{z}}$ 的平移不变性, 即

$$
E_{\bar{z}}\left[\mathrm{e}^{\mathrm{i}\langle 4 \pi \ell, \cdot\rangle} g\right](x, t)=E_{\bar{z}} g(x+4 \pi \ell, t) .
$$

因为

$$
\sum_{\ell}(1+4 \pi|\ell|)^{-N} w_{Q_{0}, \ell}(z) \lesssim w_{Q_{0}}(z),
$$

所以可推知,

$$
\left\|\left(\sum_{\nu, j}\left|\mathscr{T}_{\lambda, k}^{\nu, j} f\right|^{2}\right)^{\frac{1}{2}}\right\|_{L^{\frac{10}{3}\left(w_{Q_{k}}\right)}} \lesssim\left\|\left(\sum_{\nu}\left|E_{z_{k}}^{\nu} f_{z_{k}}\right|^{2}\right)^{\frac{1}{2}}\right\|_{L^{\frac{10}{3}\left(w_{Q_{0}}\right)}}+\operatorname{RapDec}(\lambda)\|f\|_{\frac{10}{3}} .
$$

为了完成证明, 只需把 $E_{z_{k}}^{\nu} f_{z_{k}}$ 换成与其对应的振荡积分算子 $\mathscr{T}_{\lambda}^{\nu} f$. 由 Minkowski 不等式, 有

$$
\begin{aligned}
&\left\|\left(\sum_{\nu}\left|E_{z_{k}}^{\nu} f_{z_{k}}\right|^{2}\right)^{\frac{1}{2}}\right\|_{L^{\frac{10}{3}}\left(w_{Q_{0}}\right)} \leqslant\left\|\left(\sum_{\nu}\left|E_{z_{k}}^{\nu} f_{z_{k}}\right|^{2}\right)^{\frac{1}{2}} \chi_{\left\{|z| \leqslant \lambda^{\frac{1}{2}-\frac{\varepsilon}{16}}\right\}}\right\|_{L^{\frac{10}{3}}\left(w_{Q_{0}}\right)} \\
&+\left\|\left(\sum_{\nu}\left|E_{z_{k}}^{\nu} f_{z_{k}}\right|^{2}\right)^{\frac{1}{2}} \chi_{\left\{|z| \geqslant \lambda^{\left.\frac{1}{2}-\frac{\varepsilon}{16}\right\}}\right\}}\right\|_{L^{\frac{10}{3}}\left(w_{Q_{0}}\right)} \\
& \lesssim_{N} \sum_{\ell \in \mathbb{Z}^{2}}(1+4 \pi|l|)^{-N}\left\|\left(\sum_{\nu}\left|\mathscr{T}_{\lambda}^{\nu}\left(\mathrm{e}^{\mathrm{i}\left\langle 4 \pi \ell, \partial_{x} \phi^{\lambda}\left(z_{k}, \cdot\right)\right\rangle} f\right)\left(z_{k}+v\right)\right|^{2}\right)^{\frac{1}{2}}\right\|_{L^{\frac{10}{3}}\left(w_{Q_{0}}\right)} \\
&+\operatorname{RapDec}(\lambda)\|f\|_{L^{\frac{10}{3}}}
\end{aligned}
$$

注意到情形 $\ell=0$ 是期待的估计, 我们仅需控制剩下余项. 由 (5.64) 推知,

$$
\begin{aligned}
& \sum_{\ell \in \mathbb{Z}^{2} \backslash\{0\}}\left(\frac{\pi|\ell|}{3}\right)^{-N}\left\|\left(\sum_{\nu}\left|\mathscr{T}_{\lambda}^{\nu}\left(\mathrm{e}^{\mathrm{i}\left\langle 4 \pi \ell, \partial_{x} \phi^{\lambda}\left(z_{k}, \cdot\right)\right\rangle} f\right)\left(z_{k}+v\right)\right|^{2}\right)^{\frac{1}{2}}\right\|_{L^{\frac{10}{3}}\left(w_{Q_{0}}\right)} \\
& \leqslant \sum_{\ell \in \mathbb{Z}^{2} \backslash\{0\}} \sum_{k \in \mathbb{Z}^{2} \backslash\{0\}}\left(\frac{\pi|\ell|}{3}\right)^{-N}\left(\frac{\pi|k|}{3}\right)^{-N}\left\|\left(\sum_{\nu}\left|E_{z_{k}}^{\nu} f_{z_{k}}\right|^{2}\right)^{\frac{1}{2}}\right\|_{L^{\frac{10}{3}}\left(w_{Q_{0}}((4 \pi(\ell+k), 0)+\cdot)\right)} \\
& \quad+\sum_{\ell \in \mathbb{Z}^{2} \backslash\{0\}}\left(\frac{\pi|\ell|}{3}\right)^{-N}\left\|\left(\sum_{\nu}\left|E_{z_{k}}^{\nu} f_{z_{k}}\right|^{2}\right)^{\frac{1}{2}}\right\|_{L^{\frac{10}{3}}\left(w_{Q_{0}}((4 \pi \ell, 0)+\cdot)\right)} \\
& \leqslant \frac{1}{2}\left\|\left(\sum_{\nu}\left|E_{z_{k}}^{\nu} f_{z_{k}}\right|^{2}\right)^{\frac{1}{2}}\right\| \|_{L^{\frac{10}{3}}\left(w_{Q_{0}}\right)}
\end{aligned}
$$

通过选取 $N$ 充分大可确保最后一个不等式成立. 综上所述估计可得

$$
\begin{aligned}
& \left\|\left(\sum_{\nu}\left|E_{z_{k}}^{\nu} f_{z_{k}}\right|^{2}\right)^{\frac{1}{2}}\right\|_{L^{4}\left(w_{Q_{0}}\right)} \\
& \quad \leqslant\left\|\left(\sum_{\nu}\left|\mathscr{T}_{\lambda}^{\nu} f\right|^{2}\right)^{\frac{1}{2}}\right\|_{L^{4}\left(w_{Q_{k}}\right)}+\frac{1}{2}\left\|\left(\sum_{\nu}\left|E_{z_{k}}^{\nu} f_{z_{k}}\right|^{2}\right)^{\frac{1}{2}}\right\|_{L^{4}\left(w_{Q_{0}}\right)}+\operatorname{RapDec}(\lambda)\|f\|_{L^{\frac{10}{3}}} .
\end{aligned}
$$

注意到右边 $\left\|\left(\sum_{\nu}\left|E_{z_{k}}^{\nu} f_{z_{k}}\right|^{2}\right)^{1 / 2}\right\|_{L^{4}\left(w_{Q_{0}}\right)}$ 可被左边吸收, 从而完成了 (5.55) 的证明. 


\section{4 引理 5.2 的证明}

为证明引理 5.2 , 先引入两个已知引理.

引理 $5.4^{[50]}$ 设 $\varphi$ 是支撑在以原点为中心的固定方体 $Q$ 上的光滑 bump 函数. 记 $\left\{O_{k}\right\}_{k}$ 是中 心为 $\xi_{k}$ 且具有相同尺度的正方体, $\varphi_{k}(\xi)=\varphi\left(\xi-\xi_{k}\right)$ 是从属于方体 $Q_{k}$ 的 bump 函数. 对任意函数 $f$, 有如下点态估计:

$$
\left(\sum_{k}\left|\widehat{\varphi}_{k} * f\right|^{2}\right)^{\frac{1}{2}} \leqslant C(\varphi)\left(\boldsymbol{M}\left[|f|^{2}\right]\right)^{\frac{1}{2}},
$$

其中 $\boldsymbol{M}$ 表示 Hardy-Littlewood 极大算子, 常数 $C(\varphi)$ 依赖于维数与 $\varphi$ 的有限阶导数.

第二个引理是向量值极大函数估计, 源于 Fefferman 和 Stein ${ }^{[51]}$, 或参见文献 [38].

引理 $5.5^{[51]}$ 设 $1<r, p<\infty,\left\{f_{k}\right\}_{k}$ 为函数序列, 则

$$
\left\|\left(\sum_{k}\left|\boldsymbol{M} f_{k}\right|^{r}\right)^{\frac{1}{r}}\right\|_{L^{p}\left(\mathbb{R}^{n}\right)} \leqslant C_{n} A_{p, r}\left\|\left(\sum_{k}\left|f_{k}\right|^{r}\right)^{\frac{1}{r}}\right\|_{L^{p}\left(\mathbb{R}^{n}\right)},
$$

其中

$$
A_{p, r}=\frac{r}{r-1}\left(p+\frac{1}{p-1}\right) .
$$

证明 (5.63) 的障碍在于 $w_{Q_{0}}$ 未必是 $A_{p}$ 权. 准确地说, 下面的极大函数估计:

$$
\|\boldsymbol{M} f\|_{L^{p}\left(w_{Q_{0}}\right)} \leqslant C\|f\|_{L^{p}\left(w_{Q_{0}}\right)}, \quad 1<p<\infty
$$

未必成立. 为克服该困难并保持某种局部性质, 需要进行一系列局部化. 事实上,

$$
\begin{aligned}
\left\|\left(\sum_{\nu, j}\left|E_{z_{k}}^{\nu, j} g\right|^{2}\right)^{\frac{1}{2}}\right\|_{L^{\frac{10}{3}}\left(w_{Q_{0}}\right)} \leqslant & \left\|\left(\sum_{\nu, j}\left|E_{z_{k}}^{\nu, j} g\right|^{2}\right)^{\frac{1}{2}} \chi_{\left\{|x| \leqslant \lambda^{\frac{1}{2}-\frac{\varepsilon}{4}}\right\}}\right\|_{L^{\frac{10}{3}}\left(w_{Q_{0}}\right)} \\
& +\left\|\left(\sum_{\nu, j}\left|E_{z_{k}}^{\nu, j} g\right|^{2}\right)^{1 / 2} \chi_{\left\{|x|>\lambda^{\frac{1}{2}-\frac{\varepsilon}{4}}\right\}}\right\|_{L^{10 / 3}\left(w_{B_{0}}\right)} .
\end{aligned}
$$

由于权函数在 $|z| \geqslant \lambda^{1 / 2-\varepsilon / 4}$ 之外快速衰减, 因此仅需考虑

$$
\left\|\left(\sum_{\nu, j}\left|E_{z_{k}}^{\nu, j} g\right|^{2}\right)^{\frac{1}{2}} \chi_{\left\{|x| \leqslant \lambda^{\frac{1}{2}-\frac{\varepsilon}{4}}\right\}}\right\|_{L^{\frac{10}{3}}\left(w_{Q_{0}}\right)} .
$$

固定 $t_{0}$, 考察

$$
E_{z_{k}}^{\nu, j} g\left(x, t_{0}\right)=\int \mathrm{e}^{\mathrm{i}\langle x, \eta\rangle} \rho\left(\lambda^{\frac{1}{2}-\frac{\varepsilon}{2}} \eta-j\right)\left(E_{z_{k}}^{\nu} g\right)^{\wedge}\left(\eta, t_{0}\right) d \eta,
$$

其中 $E_{z_{k}}^{\nu} g\left(x, t_{0}\right)$ 如下定义:

$$
E_{z_{k}}^{\nu} g\left(x, t_{0}\right):=\int_{\mathbb{R}^{2}} \mathrm{e}^{\mathrm{i}\left(\langle x, \eta\rangle+t_{0} h_{z_{k}}(\eta)\right)} a_{z_{k}}^{\nu}(\eta) g(\eta) d \eta .
$$

实施如下分解:

$$
E_{z_{k}}^{\nu} g\left(x, t_{0}\right)=\chi_{\left\{|x| \leqslant \lambda^{\frac{1}{2}-\frac{\varepsilon}{8}}\right\}}(x) E_{z_{k}}^{\nu} g\left(x, t_{0}\right)+\chi_{\left\{|x|>\lambda^{\left.\frac{1}{2}-\frac{\varepsilon}{8}\right\}}\right.}(x) E_{z_{k}}^{\nu} g\left(x, t_{0}\right),
$$


则只需估计

$$
\int \mathrm{e}^{\mathrm{i}\langle x, \eta\rangle} \rho\left(\lambda^{\frac{1}{2}-\frac{\varepsilon}{2}} \eta-j\right)\left(\chi_{\left\{|x| \leqslant \lambda^{\left.\frac{1}{2}-\frac{\varepsilon}{8}\right\}}\right.}(\cdot) E_{z_{k}}^{\nu} g\right)^{\wedge}\left(\eta, t_{0}\right) d \eta .
$$

事实上, 对于 $|x| \leqslant \lambda^{1 / 2-\varepsilon / 4},(5.74)$ 的第二项对于 (5.72) 的贡献可被余项控制, 即

$$
\int \frac{1}{\lambda^{1-\varepsilon}} \hat{\rho}\left(\frac{x-y}{\lambda^{\frac{1}{2}-\frac{\varepsilon}{2}}}\right) \chi_{\left\{|x|>\lambda^{\left.\frac{1}{2}-\frac{\varepsilon}{8}\right\}}\right.}(y) E_{z_{k}}^{\nu} g(y) d y \lesssim \operatorname{RapDec}(\lambda)\|g\|_{L^{10 / 3}} .
$$

我们继续估计 (5.75), 由引理 5.4 推知,

$$
\begin{aligned}
& \left(\sum_{\nu, j}\left|\int \mathrm{e}^{\mathrm{i}\langle x, \eta\rangle} \rho\left(\lambda^{\frac{1}{2}-\frac{\varepsilon}{2}} \eta-j\right)\left(\chi_{\left\{|\cdot| \leqslant \lambda^{\left.\frac{1}{2}-\frac{\varepsilon}{8}\right\}}\right.}(\cdot) E_{z_{k}}^{\nu} g\right)^{\wedge}\left(\eta, t_{0}\right) d \eta\right|^{2}\right)^{\frac{1}{2}} \\
& \quad \leqslant C\left(\sum_{\nu} \boldsymbol{M}\left[\left|\chi_{\left\{|\cdot| \leqslant \lambda^{\left.\frac{1}{2}-\frac{\varepsilon}{8}\right\}}\right.}(\cdot) E_{z_{k}}^{\nu} g\left(\cdot, t_{0}\right)\right|^{2}\right]\right)^{\frac{1}{2}} .
\end{aligned}
$$

让 $t_{0}$ 变动, 把 $(5.77)$ 代入 (5.71), 利用引理 5.5 可得

$$
\begin{aligned}
\left\|\left(\sum_{\nu, j}\left|E_{z_{k}}^{\nu, j} g\right|^{2}\right)^{\frac{1}{2}}\right\|_{L^{\frac{10}{3}}\left(w_{Q_{0}}\right)} & \lesssim\left\|\left(\sum_{\nu} \boldsymbol{M}\left[\left|\chi_{\left\{|x| \leqslant \lambda^{\left.\frac{1}{2}-\frac{\varepsilon}{8}\right\}}\right.}(\cdot) E_{z_{k}}^{\nu} g\left(\cdot, t_{0}\right)\right|^{2}\right]\right)^{\frac{1}{2}}\right\|_{L^{\frac{10}{3}}\left(w_{Q_{0}}\right)} \\
& \lesssim\left\|\left(\sum_{\nu}\left|E_{z_{k}}^{\nu} g\right|^{2}\right)^{\frac{1}{2}}\right\|_{L^{\frac{10}{3}}\left(w_{Q_{0}}\right)} .
\end{aligned}
$$

注 5.3 当一个不等式使用 Fefferman-Stein 的估计时, 会出现额外因子 $\lambda^{\varepsilon}$. 实际上, 这里使用了 如下的不等式:

$$
\left\|\sum_{m} \boldsymbol{M} g_{m}\right\|_{L^{\frac{10}{3}\left(\mathbb{R}^{3}\right)}} \lesssim_{\varepsilon} N^{\varepsilon}\left\|\sum_{m}\left|g_{m}\right|\right\|_{L^{\frac{10}{3}\left(\mathbb{R}^{3}\right)}},
$$

其中 $\#\{m\}=N, \varepsilon>0$ 任意小. 事实上, 由 Hölder 不等式以及引理 5.5 , 选取 $r^{\prime}$ 充分大, 使得 $\frac{1}{r^{\prime}} \leqslant \varepsilon$, 则有

$$
\begin{aligned}
\left\|\sum_{m} \boldsymbol{M} g_{m}\right\|_{L^{\frac{10}{3}\left(\mathbb{R}^{3}\right)}} & \leqslant\left\|\left(\sum_{m}\left|\boldsymbol{M} g_{m}\right|^{r}\right)^{\frac{1}{r}}\right\|_{L^{\frac{10}{3}\left(\mathbb{R}^{3}\right)}}(\#\{m\})^{\frac{1}{r^{\prime}}} \\
& \lesssim r r \mid\left(\sum_{m}\left|g_{m}\right|^{r}\right)^{\frac{1}{r}} \|_{L^{\frac{10}{3}\left(\mathbb{R}^{3}\right)}}(\#\{m\})^{\frac{1}{r^{\prime}}} \\
& \lesssim\left\|\sum_{m}\left|g_{m}\right|\right\|_{L^{\frac{10}{3}\left(\mathbb{R}^{3}\right)}}(\#\{m\})^{\frac{1}{r^{\prime}}}
\end{aligned}
$$

\section{5 引理 5.3 的证明}

注意到 $\operatorname{supp}_{\eta} a_{\lambda, \bar{z}}(z, \cdot) \subset B\left(\boldsymbol{e}_{2}, \varepsilon_{0}\right)$, 这里不妨假设 $\varepsilon_{0}$ 充分小. 我们可用 $f \psi$ 去替换 $f$, 其中 $\psi$ 是 支撑在 $B\left(\boldsymbol{e}_{2}, \frac{1}{5}\right)$ 上的光滑函数且在 $B\left(\boldsymbol{e}_{2}, \frac{1}{100}\right)$ 上等于 1 , 满足

$$
\left|\partial_{\eta}^{\alpha} \psi(\eta)\right| \leqslant 6^{N}, \quad \alpha \in \mathbb{N}^{2}, \quad 1 \leqslant|\alpha| \leqslant N .
$$

对 $\mathrm{e}^{\mathrm{i} \varepsilon_{\lambda}^{\overline{\bar{\lambda}}}(v, \eta)} \psi(\eta)$ 关于变量 $\eta$ 实施 Fourier 展开, 可得

$$
\mathrm{e}^{\mathrm{i} \varepsilon_{\lambda}^{\bar{z}}(v, \eta)} \psi(\eta)=\sum_{\ell \in \mathbb{Z}^{2}} b_{\ell}(v) \mathrm{e}^{\mathrm{i}\langle 4 \pi \ell, \eta\rangle},
$$


其中

$$
b_{\ell}(v)=2 \int_{Q\left(e_{2}, 1 / 4\right)} \mathrm{e}^{-\mathrm{i}\langle 4 \pi \ell, \eta\rangle} \mathrm{e}^{\mathrm{i} \varepsilon_{\lambda}^{\bar{z}}(v, \eta)} \psi(\eta) d \eta,
$$

$Q\left(\boldsymbol{e}_{2}, 1 / 4\right)$ 表示中心是 $\boldsymbol{e}_{2}$ 且边长是 $1 / 2$ 的方体. 由 (5.58) 容易看出 $\left|b_{0}(v)\right| \leqslant 1$. 分部积分, 有

$$
\left|b_{\ell}(v)\right| \leqslant 12^{N}(4 \pi|\ell|)^{-N}, \quad \text { 只要 }|v| \leqslant K, \quad \ell \neq(0,0),
$$

则得到 $(5.64)$.

至于反方向的不等式, 根据

$$
E_{\bar{z}} f_{\bar{z}}(v)=\int_{\mathbb{R}^{2}} \mathrm{e}^{\mathrm{i} \phi^{\lambda}\left(\bar{z}+v, \Psi^{\lambda}(\bar{z}, \eta)\right)} \mathrm{e}^{-\mathrm{i} \varepsilon_{\lambda}^{\bar{z}}(v, \eta)} a_{2, \bar{z}}(\eta) f \circ \Psi^{\lambda}(\bar{z}, \eta) d \eta,
$$

通过对 $\mathrm{e}^{-\mathrm{i} \varepsilon_{\lambda}^{\bar{z}}(v, \eta)}$ 关于 $\eta$ 实施 Fourier 展开, 作变量替换: $\eta \rightarrow \Psi^{\lambda}(\bar{z}, \eta)$, 得到 (5.74).

\section{6 关于高维情形的注记}

如果对相函数施加额外的凸性条件, 则命题 5.2 的高维版本也成立. 对 $2+1$ 维情形, 非零特征根 只有一个, 凸性条件是多余的. 本质上, 凸性是保证分离性条件 (4.10) 的内蕴几何条件.

设 $n \geqslant 3, a(z, \eta) \in C_{c}^{\infty}\left(\mathbb{R}^{n+1} \times \mathbb{R}^{n}\right)$ 支撑在 $B\left(0, \varepsilon_{0}\right) \times B\left(\boldsymbol{e}_{n}, \varepsilon_{0}\right)$ 上. 假设

$$
\mathcal{C}\left(\boldsymbol{e}_{2}, \varepsilon_{0}\right):=B\left(\boldsymbol{e}_{n}, \varepsilon_{0}\right) \cap \mathbb{S}^{n-1},
$$

对变量 $\eta$ 实施如下角向分解: 把 $\mathcal{C}\left(\boldsymbol{e}_{n}, \varepsilon_{0}\right)$ 分解成 $\sim N_{\lambda} \approx \lambda^{\frac{n-1}{2}}$ 个扇形区域 $\left\{\theta_{\nu}: 1 \leqslant \nu \leqslant N_{\lambda}\right\}$, 其中 每个扇形 $\theta_{\nu}$ 张成的角度为 $\approx_{\varepsilon_{0}} \lambda^{-1 / 2}$. 用 $\kappa_{\nu} \in \mathbb{S}^{1}$ 表示扇形 $\theta_{\nu}$ 的中心.

令 $\left\{\chi_{\nu}(\eta)\right\}$ 是从属于角向分解的一列齐次度为 1 的光滑截断函数, 并且构成了 $\mathbb{R}^{n} \backslash 0$ 的单位分解. 准确地讲, 满足

$$
\left\{\begin{array}{l}
\sum_{0 \leqslant \nu \leqslant N_{\lambda}} \chi_{\nu}(\eta) \equiv 1, \quad \forall \eta \in \mathbb{R}^{n} \backslash 0, \\
\left|\partial^{\alpha} \chi_{\nu}(\eta)\right| \leqslant C_{\alpha} \lambda^{\frac{|\alpha|}{2}}, \quad \forall \alpha \in \mathbb{N}^{2}, \quad|\eta|=1 .
\end{array}\right.
$$

定义

$$
\left\{\begin{array}{l}
T_{\lambda} f=\int \mathrm{e}^{\mathrm{i} \lambda \phi(z, \eta)} a(z, \eta) f(\eta) d \eta=\sum_{\nu} T_{\lambda}^{\nu} f, \\
T_{\lambda}^{\nu} f(z)=\int \mathrm{e}^{\mathrm{i} \lambda \phi(z, \eta)} a^{\nu}(z, \eta) f(\eta) d \eta,
\end{array}\right.
$$

其中 $a^{\nu}(z, \eta)=\chi_{\nu}(\eta) a(z, \eta)$. 直接验证得

$$
T_{\lambda} f=\sum_{\nu} T_{\lambda}^{\nu} f
$$

设 $n \geqslant 3$, 在类似于命题 5.2 的条件和额外的凸性条件下, 利用之前的证明方法, 可以得到

$$
\begin{aligned}
& \left\|\sum_{\nu \in \Omega} T_{\lambda}^{\nu} g \sum_{\nu^{\prime} \in \Omega^{\prime}} T_{\lambda}^{\nu^{\prime}} h\right\|_{L^{\frac{n+3}{n+1}}\left(\mathbb{R}^{n+1}\right)} \\
& \geqq_{\phi, \varepsilon} \lambda^{\frac{n-1}{2(n+3)}}\left\|\left(\sum_{\nu \in \Omega}\left|T_{\lambda}^{\nu} g\right|^{2}\right)^{\frac{1}{2}}\right\|_{L^{\frac{2(n+3)}{n+1}}\left(\mathbb{R}^{n+1}\right)}\left\|\left(\sum_{\nu^{\prime} \in \Omega^{\prime}}\left|T_{\lambda}^{\nu^{\prime}} h\right|^{2}\right)^{\frac{1}{2}}\right\|_{L^{\frac{2(n+3)}{n+1}}\left(\mathbb{R}^{n+1}\right)} .
\end{aligned}
$$


在相差余项 $\operatorname{RapDec}(\lambda)$ 的情形下, 上式意味着平方函数估计

$$
\left\|T_{\lambda} f\right\|_{L^{\frac{2(n+3)}{n+1}}\left(\mathbb{R}^{n+1}\right)} \lesssim \lambda^{\frac{n-1}{4(n+3)}}\left\|\left(\sum_{\nu}\left|T_{\lambda}^{\nu} f\right|^{2}\right)^{\frac{1}{2}}\right\|_{L^{\frac{2(n+3)}{n+1}}\left(\mathbb{R}^{n+1}\right)} .
$$

不幸的是, 我们并不能改进利用文献 [41] 中的最佳 $L^{\frac{2(n+1)}{n-1}}$ 估计和平凡 $L^{2}$ 估计插值所获得的估计. 失 败原因是光雉方向上的变系数 Kakeya 极大函数 $L^{p} \rightarrow L^{p}$ 估计的贵乏,

$$
\left\|\mathcal{M}_{\delta}\right\|_{L^{p} \rightarrow L^{p}} \leqslant C \max \left\{\left(\log \frac{1}{\delta}\right)^{\frac{1}{2}}, \delta^{-\frac{n-2}{p}}\right\}, \quad 2 \leqslant p \leqslant \infty, \quad n \geqslant 3 .
$$

众所周知, 对于 $p>2, L^{p}$-Kakeya 极大函数估计是非常困难的, 可参见文献 [17]. 综上所述, 对 $n \geqslant 3$, 在 $p \leqslant \frac{2(n+1)}{n-1}$ 的情形下, 利用双线性方法似乎很难进一步改进文献 [22] 中的结果.

\section{6 关于 Fourier 积分算子的局部光滑效应的进一步讨论}

本节考虑 Fourier 积分算子的局部光滑性估计与振荡积分算子的 $L^{p}$ 估计之间的联系. 考虑如下 振荡积分算子:

$$
T_{\lambda} f(z):=\int_{\mathbb{R}^{n-1}} \mathrm{e}^{\mathrm{i} \lambda \phi(z, \eta)} a(z, \eta) f(\eta) d \eta,
$$

其中 $\phi \in C^{\infty}\left(\mathbb{R}^{n} \times \mathbb{R}^{n-1}\right), a \in C_{c}^{\infty}\left(\mathbb{R}^{n} \times \mathbb{R}^{n-1}\right)$. 相函数满足如下 Carleson-Sjölin 条件:

$\left(\mathrm{H}_{1}\right)$ 对所有的 $(z, \eta) \in \operatorname{supp} a$, 有 $\operatorname{rank} \partial_{z \eta}^{2} \phi(z, \eta)=n-1$;

$\left(\mathrm{H}_{2}\right)$ 定义 Gauss 映射 $G: \operatorname{supp} a \rightarrow \mathbb{S}^{n-1}$ 如下: $G(z, \eta):=\frac{G_{0}(z, \eta)}{\left|G_{0}(z, \eta)\right|}$, 其中

$$
G_{0}(z, \eta):=\bigwedge_{j=1}^{n-1} \partial_{\eta_{j}} \partial_{z} \phi(z, \eta)
$$

总满足曲率条件

$$
\left.\operatorname{rank} \partial_{\eta \eta}^{2}\left\langle\partial_{z} \phi(z, \eta), G\left(z, \eta_{0}\right)\right\rangle\right|_{\eta=\eta_{0}}=n-1, \quad \forall\left(z, \eta_{0}\right) \in \operatorname{supp} a .
$$

设 $\operatorname{rank} \partial_{\eta \eta}^{2} \psi(\eta)=n-1$, 显然, (6.1) 可视为下面延拓性算子:

$$
E f(x, t)=\int_{\mathbb{R}^{n-1}} \mathrm{e}^{\mathrm{i}(x \eta+t \psi(\eta))} a(x, t, \eta) f(\eta) d \eta
$$

的推广. 类似于限制性猜想, 对于振荡积分算子 $T_{\lambda}$, Hörmander 提出了下面的猜想:

猜想 6.1 ${ }^{[40]}$ (Hörmander 猜想) 对于 $\frac{1}{q}<\frac{n-1}{2 n}, \frac{1}{q} \leqslant \frac{n-1}{n+1} \frac{1}{p^{\prime}}$, 相函数满足条件 $\left(\mathrm{H}_{1}\right)$ 和 $\left(\mathrm{H}_{2}\right)$, 则

$$
\left\|T_{\lambda} f\right\|_{L^{q}\left(\mathbb{R}^{n}\right) \lesssim \lambda^{-n / q}}\|f\|_{L^{p}\left(\mathbb{R}^{n-1}\right)} .
$$

Hörmander 猜想是非常强的. 如果 Hörmander 猜想成立, 则意味着 Bochner-Riesz 猜想和限制性 猜想成立. Hörmander [40] 证明了 $n=2$ 的情形; 对 $n \geqslant 3$, Stein ${ }^{[6]}$ 证明了 $q \geqslant \frac{2(n+1)}{n-1}$ 时的最优结果. 然 而令人惊奇的是, Bourgain ${ }^{[42]}$ 通过构造反例否定了 Hörmander 猜想, 并且指出当 $n$ 是奇数时, Stein 得到的结果是最优的. 下面是 1991 年 Bourgain 所举的反例: 
例 6.1 设相函数

$$
\varphi(x, y)=x_{1} y_{1}+x_{2} y_{2}+2 x_{3} y_{1} y_{2}+x_{3}^{2} y_{1}^{2},
$$

则当 $n=3$ 时, 对任意的 $q<4,(6.5)$ 不成立.

证明 先来验证相函数 $\varphi$ 满足条件 $\left(\mathrm{H}_{1}\right)$ 和 $\left(\mathrm{H}_{2}\right)$. 简单计算可得

$$
\frac{\partial^{2} \varphi}{\partial x \partial y}=\left(\begin{array}{ccc}
1 & 0 & 2 y_{2}+4 x_{3} y_{1} \\
0 & 1 & 2 y_{1}
\end{array}\right) .
$$

显然,

$$
\operatorname{rank} \frac{\partial^{2} \varphi}{\partial x \partial y}=2
$$

设 $\theta \in S^{2}$, 则有

$$
\begin{aligned}
& \theta \cdot \frac{\partial \varphi}{\partial x}=\theta_{1} y_{1}+\theta_{2} y_{2}+\theta_{3}\left(2 y_{1} y_{2}+2 x_{3} y_{1}^{2}\right) \\
& \frac{\partial}{\partial y}\left(\theta \cdot \frac{\partial \varphi}{\partial x}\right)=\left(\begin{array}{c}
\theta_{1}+2 \theta_{3}\left(y_{2}+2 x_{3} y_{1}\right) \\
\theta_{2}+2 \theta_{3} y_{1}
\end{array}\right) \\
& \frac{\partial^{2}}{\partial y^{2}}\left(\theta \cdot \frac{\partial \varphi}{\partial x}\right)=\left(\begin{array}{cc}
4 x_{3} & 2 \theta_{3} \\
2 \theta_{3} & 0
\end{array}\right) .
\end{aligned}
$$

如果 $\theta \neq 0$, 则 $(6.7)$ 和 (6.8) 不等于 0 . 令 $f(y)=\mathrm{e}^{\mathrm{i} \lambda} y_{2}^{2} \chi(y)$, 其中 $\chi$ 为光滑截断函数, 使得

$$
T_{\lambda} f(x)=\int_{\mathbb{R}^{2}} \mathrm{e}^{\mathrm{i} \lambda\left(x_{1} y_{1}+x_{2} y_{2}+\left(y_{2}+x_{3} y_{1}\right)\right)^{2}} a(x, y) d y .
$$

令 $\left(z_{1}, z_{2}\right):=\left(y_{1}, y_{2}+x_{3} y_{1}\right)$, 考虑曲面 $S:=\left\{x: x_{1}=x_{2} x_{3}\right\}$. 对 $x \in S$, 有

$$
T_{\lambda} f(x)=\int_{\mathbb{R}^{2}} \mathrm{e}^{\mathrm{i} \lambda\left(x_{2} z_{2}+z_{2}^{2}\right)} \tilde{a}(x, z) d z_{1} d z_{2}=\int_{\mathbb{R}} \mathrm{e}^{\mathrm{i} \lambda\left(x_{2} z_{2}+z_{2}^{2}\right)} \bar{a}\left(x, z_{2}\right) d z_{2} .
$$

相函数存在一个非退化临界点 $\left(z_{2}=-\frac{1}{2} x_{2}\right)$, 由驻相分析引理推知,

$$
\begin{cases}\left|T_{\lambda} f(x)\right| \sim \frac{1}{\sqrt{\lambda}}, & x \in \mathbb{R}^{3}, \\ \left|\nabla_{x} T_{\lambda} f(x)\right| \approx \lambda\left|T_{\lambda} f(x)\right| \lesssim \sqrt{\lambda}, & x \in \mathbb{R}^{3} .\end{cases}
$$

因此, 对 $x \in \lambda_{1 / \lambda}(S)$, 有

$$
\left|T_{\lambda} f(x)\right| \gtrsim \frac{1}{\sqrt{\lambda}}
$$

由此推出

$$
\left\|T_{\lambda} f\right\|_{q} \gtrsim \frac{C}{\sqrt{\lambda}}\left|\mathrm{N}_{1 / \lambda}(S)\right|^{\frac{1}{q}} \sim \lambda^{-\frac{1}{2}-\frac{1}{q}}, \quad\|f\|_{p} \sim C
$$

只有当 $\frac{3}{q} \leqslant \frac{1}{2}+\frac{1}{q}$, 即 $q \geqslant 4$ 时, 才会有 $\left\|T_{\lambda}\right\|_{p \rightarrow q} \lesssim \lambda^{-\frac{3}{q}}$.

当 $n$ 是偶数时, 文献 [52] 证明了 (6.5) 成立的必要条件是 $q \geqslant \frac{2(n+2)}{n}$. 在不计端点的情形下, Bourgain 和 Guth ${ }^{[53]}$ 证明了该条件还是充分的. 
定理 $6.1^{[6,53]}$ 假设算子 $T_{\lambda}$ 满足条件 $\left(\mathrm{H}_{1}\right)$ 和 $\left(\mathrm{H}_{2}\right)$, 对任意 $\varepsilon>0, \lambda \geqslant 1$,

$$
\begin{aligned}
& p \geqslant 2 \frac{n+1}{n-1}, n \text { 为奇数, } \\
& p \geqslant 2 \frac{n+2}{n}, n \text { 为偶数, }
\end{aligned}
$$

总成立

$$
\left\|T_{\lambda} f\right\|_{L^{p}\left(\mathbb{R}^{n}\right)} \lesssim \varepsilon \lambda^{-\frac{n}{p}+\varepsilon}\|f\|_{L^{p}\left(\mathbb{R}^{n-1}\right)} .
$$

简单地讲, 当 $n$ 是奇数时, $\left|T_{\lambda} f\right|$ 可能聚积在 $\frac{n+1}{2}$ 维代数簇 $Z$ 的 1 邻域内; 而当 $n$ 是偶数时, $\frac{n+1}{2}$ 不再是整数, 此时 $\left|T_{\lambda} f\right|$ 仅可能集中在 $\frac{n}{2}+1$ 维代数族 $Z$ 的 1 邻域内. 上述观察与 Kakeya 压缩现象 有关, 而在 Euclid 空间中, 如果 Kakeya 猜想成立, 则 Kakeya 压缩现象不会出现.

下面考虑 Fourier 积分算子与振荡积分算子之间的联系.

定理 6.2 ${ }^{[41]}$ 设 $\frac{2 n}{n-1} \leqslant p<\infty$, 如果对所有满足电影型曲率条件的 FIOs, 存在 $1 / p$ - 阶局部光滑 性, 则对同样指标 $p$, 以及所有满足 Carleson-Sjölin 条件的相函数 $\phi,(6.5)$ 成立.

假设相函数 $\varphi$ 满足额外的凸性假设, 即

$\left(\mathrm{H}_{3}^{\prime}\right)$ 对所有 $\left(z, \eta_{0}\right) \in \operatorname{supp} a$, 满足如下的曲率条件:

$$
\left.\operatorname{rank} \partial_{\eta \eta}^{2}\left\langle\partial_{z} \phi(z, \eta), G\left(z, \eta_{0}\right)\right\rangle\right|_{\eta=\eta_{0}}=n-1, \quad \text { 且所有特征根为正. }
$$

当 $q \geqslant \frac{2(n+2)}{n}$ 时, Lee ${ }^{[18]}$ 利用双线性方法证明了 (6.5). 随后, Bourgain 和 Guth ${ }^{[53]}$ 发展了多尺度归 纳, 利用多线性方法进一步改进了 (6.5). 最近, Guth 等 ${ }^{[49]}$ 利用多项式分解技术证明了如下最佳估计:

定理 6.3 ${ }^{[49]}$ 假设算子 $T_{\lambda}$ 满足条件 $\left(\mathrm{H}_{1}\right) 、\left(\mathrm{H}_{2}\right)$ 和 $\left(\mathrm{H}_{3}^{\prime}\right)$, 则对任意 $\varepsilon>0, \lambda \geqslant 1$,

$$
\begin{aligned}
& p \geqslant 2 \frac{3 n+1}{3 n-3}, n \text { 为奇数, } \\
& p \geqslant 2 \frac{3 n+2}{3 n-2}, n \text { 为偶数, }
\end{aligned}
$$

总有如下估计:

$$
\left\|T_{\lambda} f\right\|_{L^{p}\left(\mathbb{R}^{n}\right)} \lesssim \varepsilon \lambda^{-\frac{n}{p}+\varepsilon}\|f\|_{L^{p}\left(\mathbb{R}^{n-1}\right)} .
$$

值得注意的是, 除端点之外, 利用 $\varepsilon$ 消失性准则, 可获得不具有 $\lambda^{\varepsilon}$ 损失的估计.

对于 Fourier 积分算子而言, 若相函数满足凸性假设, 即

$\left(\mathrm{H}_{3}\right)$ 定义 Gauss 映射 $G: \operatorname{supp} a \rightarrow \mathbb{S}^{n}$ 为 $G(z, \eta):=\frac{G_{0}(z, \eta)}{\left|G_{0}(z, \eta)\right|}$, 其中

$$
G_{0}(z, \eta):=\bigwedge_{j=1}^{n} \partial_{\eta_{j}} \partial_{z} \phi(z, \eta),
$$

对所有的 $\left(z, \eta_{0}\right) \in \operatorname{supp} a$,

$$
\left.\partial_{\eta \eta}^{2}\left\langle\partial_{z} \phi(z, \eta), G\left(z, \eta_{0}\right)\right\rangle\right|_{\eta=\eta_{0}}
$$

有 $n-1$ 个正的特征根.

Riemann 流形上的波动方程 (1.6) 对应的半波算子是满足 $\left(\mathrm{H}_{3}\right)$ 的典型 Fourier 积分算子. 鉴于 Fourier 积分算子与振荡积分算子之间的联系, 有如下著名的猜想: 
猜想 6.2 设 Fourier 积分算子 $\mathscr{F} \in I^{\mu-1 / 4}$, 其中

$$
\mu<-(n-1)\left|\frac{1}{2}-\frac{1}{p}\right|+\frac{1}{p} .
$$

设相函数满足条件 $\left(\mathrm{H}_{1}\right)$ 和 $\left(\mathrm{H}_{3}\right)$, 则对任意

$$
\begin{aligned}
& p \geqslant 2 \frac{3 n+1}{3 n-3}, n \text { 为奇数, } \\
& p \geqslant 2 \frac{3 n+2}{3 n-2}, n \text { 为偶数, }
\end{aligned}
$$

有如下局部光滑性估计:

$$
\|\mathscr{F} f\|_{L^{p}\left(\mathbb{R}^{n+1}\right)} \leqslant C\|f\|_{L^{p}\left(\mathbb{R}^{n}\right)} .
$$

致谢 感谢审稿专家提出的修改建议.

\section{参考文献}

1 Chen S. Pseudodifferential Operators, 2nd ed (in Chinese). Beijing: Higher Education Press, 2006 [陈恕行. 拟微分 算子 (第 2 版). 北京: 高等教育出版社, 2006]

2 Miao C. Lectures on the Modern Harmonic Analysis and Applications (in Chinese). Beijing: Higher Education Press, 2018 [苗长兴. 现代调和分析及其应用讲义. 北京: 高等教育出版社, 2018]

3 Lax P. Asymptotic solutions of oscillatory initial value problems. Duke Math J, 1957, 24: 627-646

4 Hörmander L. Fourier integral operators. I. Acta Math, 1971, 127: 79-183

5 Stein E. Harmonic Analysis: Real-Variable Methods, Orthogonality, and Oscillatory Integrals. Princeton: Princeton University Press, 2016

6 Stein E. Oscillatory integrals in Fourier analysis. In: Beijing Lectures in Harmonic Analysis. Annals of Mathematics Studies, vol. 112. Princeton: Princeton University Press, 1986, 307-355

7 Duistermaat J, Hörmander L. Fourier integral operators. II. Acta Math, 1972, 128: 183-269

8 Qiu Q, Chen S, Shi J, et al. The Theory of Fourier Integral Operators and Its Applications (in Chinese). Beijing: Science Press, 1985 [仇庆久, 陈恕行, 是嘉鸿, 等. 傅立叶积分算子理论及其应用. 北京: 科学出版社, 1985]

9 Chen S, Qiu Q, Li C. Introduction to Paradifferential Operators (in Chinese). Beijing: Science Press, 1987 [陈恕行, 仇庆久, 李成章. 仿微分算子引论. 北京: 科学出版社, 1987]

10 Qi M. Introduction to Linear Partial Differential Operators, vol. 1 (in Chinese). Beijing: Science Press, 1986 [齐民友. 线性偏微分算子引论 (上). 北京: 科学出版社, 1986]

11 Qi M, Xu C. Introduction to Linear Partial Differential Operators, vol. 2 (in Chinese). Beijing: Science Press, 1992 [齐民友, 徐超江. 线性偏微分算子引论 (下). 北京: 科学出版社, 1992]

12 Egorov Y. The canonical transformations of pseudodifferential operators. Uspekhi Mat Nauk, 1969, 24: 235-236

13 Hörmander L. The spectral function of an elliptic operator. Acta Math, 1968, 121: 193-218

14 Maslov V. Theory of Perturbations and Asymptotic Methods. Moscow: Moscow State University Press, 1965

15 Sogge C. Hangzhou Lectures on Eigenfunctions of the Laplacian. Annals of Mathematics Studies, vol. 188. Princeton: Princeton University Press, 2014

16 Zelditch S. Eigenfunctions of the Laplacian on a Riemannian Manifold. Providence: Amer Math Soc, 2017

17 Sogge C. Fourier Integrals in Classical Analysis, 2nd ed. Cambridge Tracts in Mathematics, vol. 210. Cambridge: Cambridge University Press, 2017

18 Lee S. Linear and bilinear estimates for oscillatory integral operators related to restriction to hypersurfaces. J Funct Anal, 2006, 241: 56-98

19 Peral J. $L^{p}$ estimates for the wave equation. J Funct Anal, 1980, 36: 114-145

20 Miyachi A. On some estimates for the wave equation in $L^{p}$ and $H^{p}$. J Fac Sci Univ Tokyo Sect IA Math, 1980, 27: 331-354

21 Sogge C. Propagation of singularities and maximal functions in the plane. Invent Math, 1991, 104: 349-376

22 Mockenhaupt G, Seeger A, Sogge C. Local smoothing of Fourier integral operators and Carleson-Sjölin estimates. J Amer Math Soc, 1993, 6: 65-130

23 Wolff T. Local smoothing type estimates on $L^{p}$ for large $p$. Geom Funct Anal, 2000, 10: 1237-1288 
24 Garrigós G, Schlag W, Seeger A. Improvements in Wolff's inequality for decompositions of cone multipliers. Https: //webs.um.es/gustavo.garrigos/papers/GSS7bis.pdf

25 Garrigós G, Seeger A. On plate decompositions of cone multipliers. Proc Edinb Math Soc (2), 2009, 52: 631-651

26 Łaba I, Wolff T. A local smoothing estimate in higher dimensions. J Anal Math, 2002, 88: 149-171

27 Bourgain J, Demeter C. The proof of the $l^{2}$ decoupling conjecture. Ann of Math (2), 2015, 182: 351-389

28 Bourgain J, Demeter C, Guth L. Proof of the main conjecture in Vinogradov's mean value theorem for degrees higher than three. Ann of Math (2), 2016, 184: 633-682

29 Guth L, Wang H, Zhang R. A sharp square function estimate for the cone in $\mathbb{R}^{3}$. Ann of Math (2), 2020, 192: 551-581

30 Córdoba A. Geometric Fourier analysis. Ann Inst Fourier (Grenoble), 1982, 32: 215-226

31 Katz N, Tao T. Recent progress on the Kakeya conjecture. Publ Mat, 2000, 46: 161-179

32 Grafakos L. Modern Fourier Analysis, 2nd ed. Graduate Texts in Mathematics, vol. 250. New York-HeidelbergDordrecht-London: Springer, 2008

33 Mattila P. Fourier Analysis and Hausdorff Dimension. Cambridge: Cambridge University Press, 2015

34 Tao T. Some recent progress on the restriction conjecture. In: Fourier Analysis and Convexity. Boston: Birkhäuser, 2004, 217-243

35 Tao T. The Bochner-Riesz conjecture implies the restriction conjecture. Duke Math J, 1999, 96: 363-375

36 Wolff T. Recent work connected with the Kakeya problem. In: Prospects in Mathematics (Princeton, NJ, 1996). Providence: Amer Math Soc, 1999, 129-162

37 Wolff T. Lectures on Harmonic Analysis. Providence: Amer Math Soc, 2003

38 Grafakos L. Classical Fourier Analysis, 2nd ed. Graduate Texts in Mathematics, vol. 249. New York-HeidelbergDordrecht-London: Springer, 2008

39 Tao T, Vargas A. A bilinear approach to cone multipliers, II: Applications. Geom Funct Anal, 2000, 10: 216-258

40 Hörmander L. Oscillatory integrals and multipliers on $F L^{p}$. Ark Mat, 1973, 11: 1-11

41 Beltran D, Hickman J, Sogge C. Variable coefficient Wolff-type inequalities and sharp local smoothing estimates for wave equations on manifolds. Anal PDE, 2020, 13: 403-433

42 Bourgain J. $L^{p}$-estimates for oscillatory integrals in several variables. Geom Funct Anal, 1991, 1: 321-374

43 Wolff T. A sharp bilinear cone restriction estimate. Ann of Math (2), 2001, 153: 661-698

44 Gao C, Miao C, Yang J. Improved variable coefficient square functions and local smoothing of Fourier integral operators. arXiv:1901.01487, 2019

45 Gao C, Miao C, Yang J. Square function inequality for a class of Fourier integral operators satisfying cinematic curvature conditions. Forum Math, 2020, 32: 1375-1394

46 Lee J. A trilinear approach to square function and local smoothing estimates for the wave operator. Indiana Univ Math J, 2020, 69: 2005-2033

47 Lee S, Vargas A. On the cone multiplier in $\mathbb{R}^{3}$. J Funct Anal, 2012, 263: 925-940

48 Córdoba A. A note on Bochner-Riesz operators. Duke Math J, 1979, 46: 505-511

49 Guth L, Hickman J, Iliopoulou M. Sharp estimates for oscillatory integral operators via polynomial partitioning. Acta Math, 2019, 223: 251-376

50 Rubio de Francia J. Estimates for some square functions of Littlewood-Paley type. Publ Mat, 1983, 27: 81-108

51 Fefferman C, Stein E. Some maximal inequalities. Amer J Math, 1971, 93: 107-115

52 Wisewell L. Kakeya sets of curves. Geom Funct Anal, 2005, 15: 1319-1362

53 Bourgain J, Guth L. Bounds on oscillatory integral operators based on multilinear estimates. Geom Funct Anal, 2011, 21: $1239-1295$

\section{附录 A}

本附录详细说明如何从平方函数不等式获得局部光滑性估计, 并列举一些尚未解决的公开问题. 固定 $\bar{p}=\frac{2 n}{n-1}$. 根据 Littlewood-Paley 分解, 猜想 2.1 可归结为证明

$$
\left\|\mathrm{e}^{\mathrm{i} t \sqrt{-\Delta}} f\right\|_{L^{\bar{p}}\left(B_{R}^{n+1}\right)} \leqslant C_{\varepsilon} R^{(n-1)\left(\frac{1}{2}-\frac{1}{\bar{p}}\right)+\varepsilon}\|f\|_{L^{\bar{p}}\left(\mathbb{R}^{n}\right)}, \quad \operatorname{supp} \hat{f} \subset \mathrm{A}(1) .
$$

假设建立了如下反向平方函数不等式:

$$
\left\|\sum_{\nu} \mathrm{e}^{\mathrm{i} t \sqrt{-\Delta}} f_{\nu}\right\|_{L^{\bar{p}}\left(B_{R}^{n+1}\right)} \leqslant C_{\varepsilon} R^{\varepsilon}\left\|\left(\sum_{\nu}\left|\mathrm{e}^{\mathrm{i} t \sqrt{-\Delta}} f_{\nu}\right|^{2}\right)^{\frac{1}{2}}\right\|_{L^{\bar{p}}\left(B_{R}^{n+1}\right)},
$$


则局部光滑性估计进一步归结为证明

$$
\left\|\left(\sum_{\nu}\left|\mathrm{e}^{\mathrm{i} t \sqrt{-\Delta}} f_{\nu}\right|^{2}\right)^{\frac{1}{2}}\right\|_{L^{\bar{p}}\left(B_{R}^{n+1}\right)} \leqslant \varepsilon R^{\frac{1}{\bar{p}}+\varepsilon}\|f\|_{L^{\bar{p}}\left(\mathbb{R}^{n}\right)}
$$

为此, 用 $K_{\nu}$ 表示 $\mathrm{e}^{\mathrm{i} t \sqrt{-\Delta}} f_{\nu}$ 的核函数

$$
K_{\nu}(x, t, y)=\int_{\mathbb{R}^{n}} \mathrm{e}^{\mathrm{i}(\langle x-y, \xi\rangle+t|\xi|)} \chi_{\nu}(\xi) d \xi
$$

对任意 $N \in \mathbb{N}$, 由驻相分析技术推出

$$
\left|K_{\nu}(x, t, y)\right| \leqslant \frac{C_{N} R^{-\frac{n-1}{2}}}{\left(1+\left|(x-y) \cdot \kappa_{\nu}+t\right|+R^{-\frac{1}{2}}\left|\Pi_{\kappa_{\nu}}^{\perp}(x-y)\right|\right)^{N}},
$$

其中 $\Pi_{\kappa_{\nu}}$ 是向以 $\kappa_{\nu}$ 为法向的超平面的投影. 利用 Riesz 表示定理, 有

$$
\left\|\left(\sum_{\nu}\left|\mathrm{e}^{\mathrm{i} t \sqrt{-\Delta}} f_{\nu}\right|^{2}\right)^{\frac{1}{2}}\right\|_{L^{\bar{p}}\left(B_{R}^{n+1}\right)}^{2}=\sup _{\|g\|_{L_{x, t}^{n}\left(\mathbb{R}^{n+1}\right)=1}} \int_{\mathbb{R}^{n+1}} \sum_{\nu}\left|\mathrm{e}^{\mathrm{i} t \sqrt{-\Delta}} f_{\nu}\right|^{2} g(x, t) d x d t,
$$

由 Hölder 不等式, 可得

$$
\begin{aligned}
& \int_{\mathbb{R}^{n+1}} \sum_{\nu}\left|\mathrm{e}^{\mathrm{i} t \sqrt{-\Delta}} f_{\nu}\right|^{2} g(x, t) d x d t \\
& \quad \lesssim \int_{\mathbb{R}^{n}} \sum_{\nu}\left|f_{\nu}(y)\right|^{2} \sup _{\nu}\left|\int_{\mathbb{R}^{n+1}} K_{\nu}(x, t, y) g(x, t) d x d t\right| d y \\
& \quad \lesssim\left\|\sum_{\nu}\left|f_{\nu}\right|^{2}\right\|_{L^{\frac{n}{n-1}\left(\mathbb{R}^{n}\right)}}\left\|\sup _{\nu}\left|\int_{\mathbb{R}^{n+1}} K_{\nu}(x, t, y) g(x, t) d x d t\right|\right\|_{L^{n}\left(\mathbb{R}^{n}\right)} .
\end{aligned}
$$

为了完成证明, 需要建立两个不等式, 其一是平方函数不等式

$$
\left\|\left(\sum_{\nu}\left|f_{\nu}\right|^{2}\right)^{\frac{1}{2}}\right\|_{L^{p}\left(\mathbb{R}^{n}\right)} \lesssim R^{\varepsilon}\|f\|_{L^{p}\left(\mathbb{R}^{n}\right)}, \quad 2 \leqslant p \leqslant \frac{2 n}{n-1} .
$$

当 $n=2$ 时, Córdoba ${ }^{[30]}$ 证明了 (A.7), 但是高维情形仍是公开的. 其二是极大 Nikodym 函数, 事实上,

$$
\left\|\sup _{\nu}\left|\int_{\mathbb{R}^{n+1}} K_{\nu}(x, t, y) g(x, t) d x d t\right|\right\|_{L^{n}\left(\mathbb{R}^{n}\right)} \lesssim_{\varepsilon} R^{1-\frac{1}{n}+\varepsilon} .
$$

(A.8) 是下面 Nikodym 极大函数猜想的直接推论.

猜想 A.1 设

$$
\mathcal{T}_{\nu}:=\left\{(x, t): x \in B_{R}^{n}(0), 0 \leqslant t \leqslant R,\left|x \cdot \kappa_{\nu}+t\right| \leqslant 1,\left|\Pi_{\kappa_{\nu}}^{\perp}(x)\right| \leqslant R^{\frac{1}{2}}\right\}
$$

则

$$
\left(\int_{\mathbb{R}^{n}} \sup _{\nu}\left|\frac{1}{\left|\mathcal{T}_{\nu}\right|} \int_{\mathcal{T}_{\nu}} g(y-x, t) d x d t\right|^{n} d y\right)^{\frac{1}{n}} \lesssim_{\varepsilon} R^{-\frac{1}{n}+\varepsilon}\|g\|_{L^{n}\left(\mathbb{R}^{n+1}\right)} .
$$

迄今为止, Mockenhaupt 等 ${ }^{[22]}$ 证明了 $n=2$ 对应的最优结果, 然而, 高维损失太多, 与期待的猜 想尚有相当的差距. 


\section{Local smoothness of Fourier integral operators and related research}

\section{Chuanwei Gao \& Changxing Miao}

Abstract In this survey, we are devoted to reviewing local smoothing estimates and the related research of Fourier integral operators satisfying the cinematic curvature conditions, which contain non-degenerate conditions and curvature conditions. We focus on how to establish a variable coefficient version of the square function inequality through the bilinear method, and then improve the local smoothing estimates established by MockenhauptSeeger-Sogge. At the same time, the difficulty and the possible ways of solving the local smoothing conjecture, and connections with other well-known mathematical conjectures are also discussed.

Keywords Fourier integral operator, local smoothing estimates, square function inequality, bilinear method, cinematic curvature condition

MSC(2020) 35S30, 35L15

doi: $10.1360 /$ SSM-2020-0173 